\title{
A Hybrid Clustering-Based Approach to Color Modification of Art Paintings for Protanopes and Deuteranopes
}

\author{
Emmanouil Mavrikos $^{\circledR}$, Nikolaos Melissaris, George Tsekouras* ${ }^{*}$ \\ Intelligent Systems Research Laboratory, Department of Cultural Technology and Communication, University of the Aegean, \\ Mytilene, Lesvos Island, Greece \\ Email: gtsek@ct.aegean.gr
}

Received: 06 November 2021; Revised: 31 December 2021; Accepted: 14 January 2022

\begin{abstract}
The normal human color vision is trichromatic, and it originates from the comparison of the rates at which photons are absorbed by three types of photoreceptor cone-cells contained in the eye's retina. The absence or malfunctioning of one or two types of cone results in color-blindness. This paper proposes an algorithmic framework to appropriately modify (i.e., recolor) art paintings for two types of color-blindness called protanopia and deuteranopia. The algorithmic framework employs four distinct steps applied in sequence. First, the image colors are clustered into a prespecified number of representative colors. The second step determines the representative colors that are confused by the protanopes or the deuteranopes. Third, an optimization problem is proposed to appropriately recolor the representative colors detected in the previous step. Finally, given the recolored representative colors, the original image pixels associated with those colors are also modified accordingly. The method is tested and evaluated in terms of quantitative, qualitative, and subjective comparison with three other recoloring algorithms. The results are promising in the sense that the proposed method outperforms the competitive algorithms, maintaining the overall aesthetic of the paintings.
\end{abstract}

Keywords: color-blindness, cluster analysis, image recoloring, art paintings, optimization

\section{Introduction}

The human color vision is based on three types of photoreceptor cone-cells called the L, M, and S cones, which define the LMS color space [1-3]. The L-cones correspond to red, the M-cones to green, and the S-cones to blue [3-4]. The respective spectral sensitivities are shown in Figure 1.

Color-blindness is the inability to correctly perceive colors and is caused by the absence or the dysfunctionality of one or two types of cone [4-5]. The most severe type of color-blindness is achromatopsia, which is caused by the absence of two types of cones [6]. The absence of one type of cones causes color vision deficiency (CVD) known as dichromacy [5-7]. Dichromacy comes in three types namely protanopia (i.e., the L-cones are missing), deuteranopia (i.e., the M-cones are missing), and tritanopia (i.e., the S-cones are missing) [6-9]. Finally, the third category of colorblindness is anomalous trichromacy, which is the most common one. It is divided into the protanomaly (i.e., the L-cones are malfunctioning), the deuteranomaly (i.e., the M-cones are malfunctioning), and the tritanomaly (i.e., the S-cones are malfunctioning).

Copyright (C2022 George Tsekouras, et al.

DOI: https://doi.org/10.37256/ccds.3120221235

This is an open-access article distributed under a CC BY license

(Creative Commons Attribution 4.0 International License)

https://creativecommons.org/licenses/by/4.0/ 


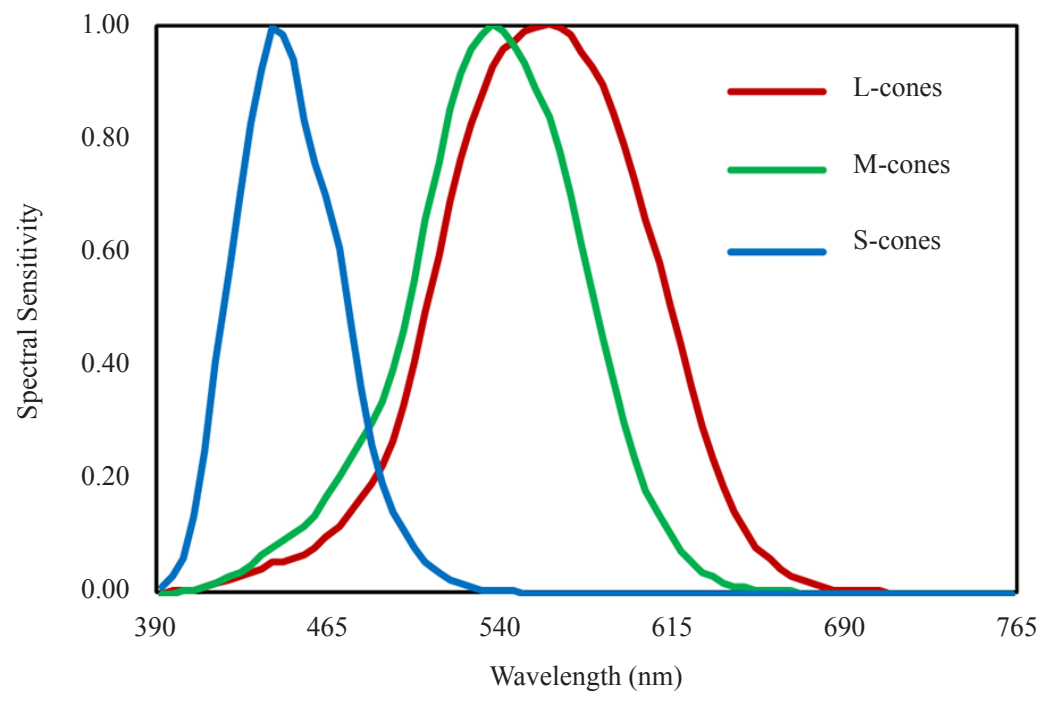

Figure 1. The cones' spectral sensitivities [4]

Image recoloring for color-blindness is based on using appropriately designed image processing tools to modify the color and enhance the perception of the color-blind. In general, the implementation of such kinds of methodologies is carried out subject to certain criteria such as the color naturalness and the enhancement of color contrast $[5,8,10]$. Naturalness concerns the preservation of the color difference, as perceived by the color-blind, between the original and the modified image in terms of their aesthetic similarity. On the other hand, contrast enhancement is vital for the colorblind to perform effective object recognition.

In this paper, a novel approach is developed to modify the color of digitized art paintings and improve the color perception for protanopia and deuteranopia. As shown in Figure 1 the spectral sensitivities in these two cases appear to be highly overlapped. Therefore, the effects are similar, and these two cases are referred to as red-green color vision deficiency [9]. The proposed approach is based on maintaining a natural appearance of the recolored (i.e., modified) paintings. However, this endeavor is challenging because of the complexity of such kinds of images $[6,8,11]$. At this point, it has to be pointed out that many color-blind people are at a disadvantage when choosing to study or enjoy art paintings because of certain difficulties in recognizing semantic objects $[8,11]$. Therefore, developing methodologies to improve the accessibility of the color-blind in cultural content (e.g., paintings) is an important issue [11-14].

The proposed methodology consists of four main steps, which are briefly described next. The first step focuses on grouping the original image's colors into a pre-specified number of clusters. To accomplish this task, a hybrid clustering algorithm, that combines crisp and fuzzy clustering, is used to obtain a partition of the input image colors into a number of clusters. The resulting clusters possess a hybrid structure, where crisp and fuzzy areas coexist. The presence of crisp and fuzzy areas is expected to provide flexibility in deciding which image colors must be recolored or not. Second, a standard algorithm is used to provide the dichromatic simulation of the resulting cluster centers and to detect the centers that are confused by the dichromats. Third, the confusing cluster centers are recolored in terms of a specialized daltonization process, which minimizes an objective function that involves the contrast enhancement criterion and a regularization part, which quantifies the preservation of naturalness. Fourth, the algorithm appropriately modifies the input image colors, which are assigned to the cluster centers that were recolored in the previous step. That modification is conducted by an interpolation-based mechanism.

The rest of the paper is synthesized as follows. Section 2 discusses related work and the current contribution. Section 3 analytically presents the hybrid clustering approach. The proposed recoloring algorithm is described in Section 4. Section 5 depicts the experimental study, and the paper concludes in Section 6. 


\section{Related studies and the current contribution}

So far, several methodologies have been developed to address the problem of image recoloring to alleviate the effects of dichromacy. Tsekouras et al. [6] used a set of confusion lines in the XYZ color space to appropriately relocate confusing colors to different positions so that can be easily distinguished by the color-blind. In [7], Lin et al. performed image recoloring using an opponent color space where they separated colors in terms of eigenvector processing. Chatzistamatis et al. [8] developed a deep learning network to identify semantic image information and then used it to efficiently modify certain areas of the image. In [10], the image recoloring is conducted in the XYZ color space, where color normalization, angular color rotation, and color de-normalization are applied in sequence. Choi et al. [15] proposed a confusion-line-based algorithm to translate the minimum number of color regions into optimal colors using a confusion line database to quantify the colors not distinguished by the color-blind. The algorithm introduced in [16] performs the recoloring process in terms of a mass-spring optimization to preserve the original image luminance.

Wang et al. [17] proposed a recoloring method, employing an optimized dichromacy projection for compensating color-blindness by developing an algorithmic framework to calculate the weight of naturalness and contrast. Two algorithms were utilized to solve the optimization problem, both based on the projected gradient descent and discrete search approach. In [18] the color differences as perceived by dichromats were quantified and an optimization approach was carried out to minimize those differences subject to certain contrast constraints. Kovalev and Petrou [19] minimized the differences between colors as seen by the normal viewer and color-blind using appropriately defined similarities in the CIELab color space, where the optimization was performed in terms of a large image database applying a randomized greedy algorithm. Similarly, in [20] Zhu et al. employed a recoloring approach for red-green dichromats, implemented in the $\mathrm{L}^{*} \mathrm{a}$ b space, preserving global and local contrast, and naturalness criteria. They introduced a color gamut for dichromats in the $\mathrm{L}^{*} \mathrm{a}^{*} \mathrm{~b}$ space, considering only the changes in the chromatic components $\mathrm{a}^{*}$ and $\mathrm{b}^{*}$, because the luminance of the arbitrary color should be preserved.

In [21], the color discrimination was assisted by a color transformation process that maps the normal color vision space to the respective dichromat color vision space. The methodology proposed in [22] introduced an integrated portable video system able to assist color-blind people in object discrimination. The system used an input video sequence, which can be adapted to fit each user's visual model compensating the perceptual defects and reducing the color information by modifying the colors of a scene so that they become distinguishable by the color-blind. Ma et al. [23] employed a self-organizing map to generate nonlinear color maps that retained the relationships between neighboring colors. The use of generative adversarial networks (GANs) was discussed by Li et al. in [24], where a self-adapting recoloring method was proposed. Specifically, the authors used an improved tree-based quantification method to obtain an optimal set of key colors of the original image. Then, a screening tool for datasets, related to color-blindness, was designed to integrate multiple recoloring methods.

$\mathrm{Xu}$ et al. [25] proposed a statistical approach based on confidence intervals according to the differences between key colors, which were extracted in the RGB space applying the $k$-means clustering. Key color confidence is introduced to judge and recolor unrecognizable colors sequentially, which improves the computational time significantly. Differences between one color and other colors with higher confidence are taken as the unrecognizable benchmark for this color.

Bonacic et al. [26] implemented an algorithmic framework to adapt interfaces using ontologies. The constructed ontology formally represents knowledge about dichromacy, personal preferences, and recoloring algorithms providing the means to develop adaptable web interfaces to meet color-blind users' individual needs. Chin and Sabudin in [27] performed a transformation of website images in the RGB space, where confusing color areas are appropriately mapped onto areas that can be easily discriminated by dichromats. In [28], combinations of confusing colors were extracted using a measure of color differences that quantified their effect on dichromats. In [29], Huang et al. generated a set of key colors and determined a transformation able to maintain the contrast between pairs of those colors. Finally, Li et al. [30] proposed a saliency-based image correction method that performs color correction on the salient region of the image to succeed saliency correction for color-blind images. An adversarial network is trained to transform the corrected image into an appropriately recolored image.

In this paper, a novel approach is proposed to modify/enhance a color image for protanopes and deuteranopes. In relation to the above-mentioned algorithms, the main contribution of the current methodology concerns the way the naturalness and contrast enhancement criteria are handled. In this regard, the novelty of the method is summarized as 
follows:

- The naturalness criterion is considered in terms of a two-fold procedure. First, the use of the hybrid clustering algorithm reduces the number of colors to be elaborated by combining the merits of the well-known $k$-means and fuzzy $k$-means methods. In this direction, the cluster centers confused by the color-blind are detected and the method modifies only the pixels that were assigned to those centers. Therefore, the number of pixels to be recolored is only a fraction of the total number of the original image's pixels. Second, an appropriate naturalness index is considered as the regularization part of a specially designed objective function.

- The contrast enhancement criterion is fulfilled in terms of the above-mentioned objective function, which involves the confusing and non-confusing cluster centers along with their color-blind simulations as well.

\section{Hybrid clustering}

Two well-known clustering approaches are the standard $k$-means and the fuzzy $k$-means algorithms. Both appear to have certain limitations and advantages, as well. The main difficulty involved in the implementation of the $k$-means algorithm is the strong dependency on initialization, which guides the optimization process to find inferior local minima due to the "winner takes all" property [31]. As such, the resulting partition of the data set is underutilized in the sense that some clusters are oversized while other clusters are undersized or, depending on the problem, they do not contain any data at all. However, the $k$-means is a very fast algorithm, yielding very low computational costs, and therefore, it is capable of dealing with big data.

On the other hand, the fuzzy $k$-means appear to have the opposite behavior. The presence of fuzziness provides a soft competition property, which forces all cluster centers to move from their initial positions [32]. Thus, they become competitive with one another, and the resulting data partition is not underutilized avoiding undesired local minima. However, the implementation of the fuzzy $k$-means is a computationally complex process, yielding a slow optimization process [32-33].

Given a set of $q$-dimensional data $X=\left.\left\{\boldsymbol{x}_{k}\right\}\right|_{k=1} ^{N}$, with $\boldsymbol{x}_{k} \in R^{q}$, the $k$-means $(k M)$ minimizes the following objective function,

$$
J_{k M}=\sum_{k=1}^{N} \sum_{i=1}^{n} u_{i k}\left\|\boldsymbol{x}_{k}-\boldsymbol{v}_{i}\right\|^{2}
$$

where $n$ is the number of clusters, $u_{i k} \in\{0,1\}$ the crisp membership degree of $\boldsymbol{x}_{k}$ to the $i$-th cluster, and $\boldsymbol{v}_{i} \in R^{q}$ is the center element of the $i$-th cluster.

Relationally, the task of the fuzzy $k$-means $(F k M)$ is to minimize the subsequent objective function,

$$
J_{F k M}=\sum_{k=1}^{N} \sum_{i=1}^{n}\left(u_{i k}\right)^{2}\left\|\boldsymbol{x}_{k}-\boldsymbol{v}_{i}\right\|^{2}
$$

where the symbols are the same as above. Note that in the case of $F k M, u_{i k} \in[0,1]$ is the fuzzy membership degree of the $i$-th cluster. The minimization of (2) is carried out under the constraint,

$$
\sum_{i=1}^{n} u_{i k}=1
$$

In [34], we have developed a hybrid clustering approach that combines the $k$-means and the fuzzy $k$-means in a unified framework, which utilizes the merits of the two algorithms. The basic task of the present endeavor employs that method to perform the cluster analysis. The objective function used comes in the form, 


$$
J_{H}=\lambda \sum_{k=1}^{N} \sum_{i=1}^{n} u_{i k}\left\|\boldsymbol{x}_{k}-\boldsymbol{v}_{i}\right\|^{2}+(1-\lambda) \sum_{k=1}^{N} \sum_{i=1}^{n}\left(u_{i k}\right)^{2}\left\|\boldsymbol{x}_{k}-\boldsymbol{v}_{i}\right\|^{2}
$$

The problem is to minimize the function $J_{H}$ under the constraint in Eq. (3). We can easily show that the membership degrees and the cluster centers that solve the above-mentioned optimization problem are respectively given as [34],

$$
\begin{aligned}
& u_{i k}=\frac{2+(n-2) \lambda}{2(1-\lambda)}\left[\sum_{j=1}^{n}\left(\frac{\left\|\boldsymbol{x}_{k}-\boldsymbol{v}_{i}\right\|}{\left\|\boldsymbol{x}_{k}-\boldsymbol{v}_{j}\right\|}\right)^{2}\right]^{-1}-\frac{\lambda}{2(1-\lambda)} \\
& \boldsymbol{v}_{i}=\frac{\sum_{k=1}^{N}\left(\lambda u_{i k}+(1-\lambda)\left(u_{i k}\right)^{2}\right) \boldsymbol{x}_{k}}{\sum_{k=1}^{N}\left(\lambda u_{i k}+(1-\lambda)\left(u_{i k}\right)^{2}\right)}
\end{aligned}
$$

with $\lambda \in(0,1)$. Then, the algorithm is based on applying in sequence the above two equations. This is accomplished by the subsequent methodology.

Eq. (5) directly implies that it is possible to obtain negative membership degrees. Indeed, this is true when the next inequality is satisfied,

$$
\sum_{j=1}^{n}\left(\frac{\left\|\boldsymbol{x}_{k}-\boldsymbol{v}_{i}\right\|}{\left\|\boldsymbol{x}_{k}-\boldsymbol{v}_{j}\right\|}\right)^{2} \geq \frac{2+(n-2) \lambda}{\lambda}
$$

Since negative membership degrees have no physical meaning, it is natural to assume that whenever (7) is true, the data vector $\boldsymbol{x}_{k}$ is not affected by the cluster center $\boldsymbol{v}_{i}$. The set of clusters centers that affect the vector $\boldsymbol{x}_{k}$ is defined as

$$
\Delta_{k}=\left\{v_{i}: u_{i k}>0\right\}
$$

and its cardinality is denoted as $\left|\Delta_{k}\right|$. As the learning process proceeds, the number of cluster centers that affect the data vector $\boldsymbol{x}_{k}$ reduces, which means that $\Delta_{k}$ reduces in size, also. In the $t$-th iteration, eq. (8) is modified as follows,

$$
\Delta_{k}^{(t)}=\left\{v_{i} \in \Delta_{k}^{(t-1)}: \sum_{v_{j} \in \Delta_{k}^{(t-1)}}\left(\frac{\left\|\boldsymbol{x}_{k}-\boldsymbol{v}_{i}\right\|}{\left\|\boldsymbol{x}_{k}-\boldsymbol{v}_{j}\right\|}\right)^{2}<\frac{2+\left(\left|\Delta_{k}^{(t-1)}\right|-2\right) \lambda}{\lambda}\right\}
$$

If a cluster center does not belong to $\Delta_{k}^{(t)}$ the respective membership degree is set equal to zero, while if it belongs to the set $\Delta_{k}^{(t)}$ the corresponding membership degree is calculated by modifying eq. (5) as,

$$
u_{i k}^{(t)}=\frac{2+\left(\left|\Delta_{k}^{(t)}\right|-2\right) \lambda}{2(1-\lambda)}\left[\sum_{\boldsymbol{v}_{j} \in \Delta_{k}^{(t)}}\left(\frac{\left\|\boldsymbol{x}_{k}-\boldsymbol{v}_{i}\right\|}{\left\|\boldsymbol{x}_{k}-\boldsymbol{v}_{j}\right\|}\right)^{2}\right]^{-1}-\frac{\lambda}{2(1-\lambda)}
$$


It is possible that the sum of membership degrees for a specific data vector $\boldsymbol{x}_{k}$ will not be equal to 1, violating the constraint in Eq. (3). In that case we normalize the membership degrees as follows,

$$
\hat{u}_{i k}^{(t)}=\frac{u_{i k}^{(t)}}{\sum_{i=1}^{n} u_{i k}^{(t)}}
$$

In (11) we considered all cluster centers because those that do not belong to $\Delta_{k}^{(t)}$ have zero membership degree and their influence is zero. Finally, the cluster centers are updated as,

$$
\boldsymbol{v}_{i}^{(t)}=\frac{\sum_{k=1}^{N}\left(\lambda \hat{u}_{i k}^{(t)}+(1-\lambda)\left(\hat{u}_{i k}^{(t)}\right)^{2}\right) \boldsymbol{x}_{k}}{\sum_{k=1}^{N}\left(\lambda \hat{u}_{i k}^{(t)}+(1-\lambda)\left(\hat{u}_{i k}^{(t)}\right)^{2}\right)}
$$

As the learning goes on, some sets $\Delta_{k}$ will contain only one cluster center. In this case, the respective data vector is transferred in the crisp mode, otherwise its stays in fuzzy mode. Thus, the obtained clusters contain crisp and fuzzy areas yielding a hybrid partition of the original data set [34]. To this end, the hybrid clustering algorithm is given by iteratively applying (9), (10), (11), and (12). For further details, the interested reader is referred to [34].

Finally, it is noted that the transfer rate from fuzzy to crip mode depends on the value of the parameter $\lambda$. In this paper, to obtain a tradeoff between the crisp and fuzzy effects, we select $\lambda=0.5$.

\section{The proposed image recoloring for dichromats}

The proposed method concerns the two important cases of dichromacy namely, protanopia and deuteranopia. The color spaces involved are the RGB, XYZ, LMS color spaces. These three spaces are denoted as $S_{R G B}, S_{X Y Z}$ and $S_{L M S}$. The transformations between those spaces are denoted as: $\varphi: S_{R G B} \rightarrow S_{X Y Z}, \theta: S_{X Y Z} \rightarrow S_{L M S}$, and $h: S_{R G B} \rightarrow S_{L M S}$.

In [35-36], Vienot et al. showed that the color space seen by protanopes and deuteranopes is a proper subspace of the $S_{R G B}$. We denote the protanope subspace as $\Phi_{p r} \subset S_{R G B}$ and the deuteranope subspace as $\Phi_{d t} \subset S_{R G B}$. They employed matrix-based mappings to define the transformations $\varphi$ and $\theta$, in order to develop an appropriate form of the transformation $h$, which means that the transformation $h$ was carried out in terms of the transformations $\varphi$ and $\theta$. Finally, using the inverse transform $h^{-1}: S_{L M S} \rightarrow S_{R G B}$, they defined the mappings from the space $S_{R G B}$ to the subspaces $\Phi_{p r}$ and $\Phi_{d t}, f_{p r}: S_{R G B} \rightarrow \Phi_{p r}$ and $f_{d t}: S_{R G B} \rightarrow \Phi_{d t}$. Thus, given a color $\boldsymbol{p} \in S_{R G B}$ the above mappings yield $\boldsymbol{p}_{p r}=f_{p r}(\boldsymbol{p}) \in \Phi_{p r}$ and $\boldsymbol{p}_{d t}=f_{d t}(\boldsymbol{p}) \in \Phi_{d t}$. For further details, the interested reader is referred to [35-36].

From now on the subspaces $\Phi_{p r}, \Phi_{d t}$, and the mappings $f_{p r}, f_{d t}$ are symbolized as $\Phi_{D}$ and $f_{D}$ where $D$ denotes the protanopia or deuteranopia effects, depending on which dichromacy case we are referring to.

Let us assume that we are given a color image $\boldsymbol{I}=\left[\boldsymbol{p}_{q_{1} q_{2}}\right]$ with size $M_{1} \times M_{2}$, where $\boldsymbol{p}_{q_{1} q_{2}} \in S_{R G B}$ is an image pixel, with $1 \leq q_{1} \leq M_{1}, 1 \leq q_{2} \leq M_{2}$. The proposed approach consists of several steps applied in sequence.

The first step concerns the implementation of the hybrid clustering process described in the previous section to cluster the colors of the input image $\boldsymbol{I}$. To reduce the computational cost, the appropriate number of clusters is preselected as

$$
n=\gamma \sqrt{\frac{M_{1} M_{2}}{M_{1}+M_{2}}}
$$


where $\gamma$ is a factor to produce a relatively small number of clusters for a certain image size. In this paper, it is selected $\gamma$ $=0.5$.

In the second step, the generated clusters centers are denoted as $V=\left\{\boldsymbol{v}_{1}, \boldsymbol{v}_{2}, \ldots, \boldsymbol{v}_{n}\right\}$. Each one of these cluster centers is transformed into the corresponding dichromacy simulated-color $f_{D}\left(\boldsymbol{v}_{i}\right), i=1,2, \ldots, n$. By selecting an integer number $\delta<<255$, we check the next condition [8,11],

$$
\left\|v_{i}-f_{D}\left(v_{i}\right)\right\| \leq \delta
$$

If the above condition is true then the dichromatic perception of the color $\boldsymbol{v}_{i}$ is not much different than the normal color vision, and the dichromat is able to distinguish it. Therefore, this color will remain intact. On the other hand, if the condition is then the color $\boldsymbol{v}_{i}$ is confused by the dichromat, and therefore it should be appropriately recolored. In this paper, we select $\delta=30$ [6]. Thus, the set $V$ is divided into two disjoint subsets $\Omega_{1}$ and $\Omega_{2}$. The set $\Omega_{1}=\left\{\boldsymbol{r}_{1}, \boldsymbol{r}_{2}, \ldots\right.$, $\left.\boldsymbol{r}_{n_{1}}\right\} \subset V$ contains all cluster centers confused by the dichromat and must be modified/recolored, and the set $\Omega_{2}=\left\{\boldsymbol{o}_{1}\right.$, $\left.\boldsymbol{o}_{2}, \ldots, \boldsymbol{o}_{n_{2}}\right\} \subset V$ that contains all the cluster centers that remain intact, such that $\Omega_{1} \cup \Omega_{2}=V$, and $\Omega_{1} \cap \Omega_{2}=\varnothing$. The dichromatic simulations for the elements of the above sets are

$$
\begin{gathered}
\boldsymbol{r}_{i, D}=f_{D}\left(\boldsymbol{r}_{i}\right), i=1,2, \ldots, n_{1} \\
\boldsymbol{o}_{j, D}=f_{D}\left(\boldsymbol{o}_{j}\right), j=1,2, \ldots, n_{2}
\end{gathered}
$$

The third step implements the methodology developed in [8] to obtain the recoloring of the elements of $\Omega_{1}$, denoted as $\boldsymbol{r}_{i, \text { rec }}\left(i=1,2, \ldots, n_{1}\right)$. The difference vector between a color that belongs to $\Omega_{1}$, and its corresponding dichromatic simulation is:

$$
\operatorname{dif}\left(\boldsymbol{r}_{i}, \boldsymbol{r}_{i, D}\right)=\boldsymbol{r}_{i}-\boldsymbol{r}_{i, D}
$$

To generate the colors $\boldsymbol{r}_{i, \text { rec }}\left(i=1,2, \ldots, n_{1}\right)$ we employ the algorithm developed in $[8,11]$,

$$
\boldsymbol{r}_{i, \mathrm{rec}}=\boldsymbol{r}_{i}+\boldsymbol{A}_{i} \operatorname{dif}\left(\boldsymbol{r}_{i}, \boldsymbol{r}_{i, D}\right), i=1,2, \ldots, n_{1}
$$

where

$$
\boldsymbol{A}_{i}=\left[\begin{array}{ccc}
\alpha & \mu_{i, 12} & 0 \\
\mu_{i, 21} & \beta & 0 \\
\mu_{i, 31} & \mu_{i, 32} & 1
\end{array}\right], i=1,2, \ldots, n_{1}
$$

Note that for each cluster there exists one matrix $\boldsymbol{A}_{i}$. In the $S_{R G B}$ space, the first row of $\boldsymbol{A}_{i}$ corresponds to the $R$-part, the second to the $G$-part, and the third to the $B$-part of the $R G B$ color. The application of eq. (18) attempts to decrease the effects of coordinates $R$ and $G$ in favor of the coordinate $B$. For the protanopia case, this strategy obtains less saturated red/oranges and more saturated greens, increasing the contrast and therefore, decreasing color confusion [8, 11]. On the other hand, for the deuteranopia case, it obtains a reduction of the green color in favor of red and blue colors yielding similar results $[8,11]$. The dichromacy simulation of $\boldsymbol{r}_{i, r e c}$ is calculated as:

$$
\boldsymbol{r}_{i, r e c, D}=f\left(\boldsymbol{r}_{i, r e c}\right)
$$


Regarding the matrix $\boldsymbol{A}_{i}$, in the protanopia case the parameter $\alpha$ is fixed in $\alpha \in[-0.5,0], \beta=1, \mu_{i, 21}, \mu_{i, 31} \in(0,1)$, and $\mu_{i, 12}, \mu_{i, 32}=0$, for $i=1,2, \ldots, n_{1}$. In the deuteranopia case we select $\alpha=0$, the parameter $\beta$ is fixed in $\beta \in[-0.5,0]$, $\mu_{i, 21}=\mu_{i, 31}=0$, and $\mu_{i, 12}, \mu_{i, 32} \in(0,1)$, for $i=1,2, \ldots, n_{1}$.

The main target of our approach is to estimate appropriate values of the parameters $\mu_{i, 21}, \mu_{i, 31}$ for protanopia, and $\mu_{i, 12}, \mu_{i, 32}$ for deuteranopia case.

To accomplish that task, we employ the objective function developed in [8], and adjust it to meet the requirements of the present contribution as follows,

$$
E=E_{1}+E_{2}+\varepsilon E_{3}
$$

where

$$
\begin{aligned}
& E_{1}=\frac{1}{n_{1} n_{2}} \sum_{i=1}^{n_{1}} \sum_{j=1}^{n_{2}}\left\|\boldsymbol{r}_{i}-\boldsymbol{o}_{j}\right\|-\left\|\boldsymbol{r}_{i, r e c, D}-\boldsymbol{o}_{j, D}\right\| \| \\
& E_{2}=\frac{1}{n_{1}^{2}} \sum_{i=1}^{n_{1}} \sum_{j=1}^{n_{1}}\left\|\boldsymbol{r}_{i}-\boldsymbol{r}_{j}\right\|-\left\|\boldsymbol{r}_{i, r e c, D}-\boldsymbol{r}_{j, r e c, D}\right\| \\
& E_{3}=\frac{1}{n_{1}} \sum_{i=1}^{n_{1}}\left\|\boldsymbol{r}_{i}-\boldsymbol{r}_{i, r e c}\right\|
\end{aligned}
$$

The minimization of $E_{1}$ attempts to keep the contrast between confusing colors and colors that remain intact, as they are perceived by a dichromat, close to the respective contrast as seen by a normal color vision viewer [8, 11]. The minimization of $E_{2}$ attempts to keep the contrast between the recolored colors as seen by a dichromat close to the respective contrast of the original colors as seen by a normal color vision viewer $[8,11]$. The minimization of $E_{3}$ attempts to keep the recolored colors close to the original ones, maintaining a natural appearance of the image. Finally, the parameter $\varepsilon$ in eq. (21) is a regularization parameter that controls the effect of the error $E_{3}$ on the optimization result.

Since, for each dichromacy case, we have $n_{1}$ cluster centers that must be recolored and each one of them is associated with two parameters in the corresponding matrix (given in eq. (19)), the number of parameters involved in the optimization problem are $2 n_{1}$ for protanopia and $2 n_{1}$ for deuteranopia.

To carry out the minimization of $E$ we use the differential evolution (DE) algorithm [37]. There are three major algorithmic stages involved in the application of the DE algorithm: (a) mutation, (b) crossover, and (c) selection. The parameters used to carry out the above stages are defined as follows: the parameter $F r \in(0,1]$ controls the rate of the evolutionary process, and the parameter $C r \in[0,1]$ controls the number of features passed in the next generation. Herein, the number of particles is denoted as $D_{\text {particles }}$, while the optimization runs for $t_{\max }$ iterations. Based on the above analysis it follows that the dimensionality of the feature space is equal to $2 n_{1}$ for both protanopia and deuteranopia. To this end, the implementation of DE yields the parameters of the matrices $\boldsymbol{A}_{i}$, which are then used in eq. (18) to estimate the recolored colors $\boldsymbol{r}_{i, \text { rec }}\left(i=1,2, \ldots, n_{1}\right)$.

Finally, the fourth step concerns the recoloring of the image pixels that belong to the clusters associated with the centers $\left\{\boldsymbol{r}_{1}, \boldsymbol{r}_{2}, \ldots, \boldsymbol{r}_{n_{1}}\right\}$. Specifically, the image pixel $\boldsymbol{p}_{i j}$ assigned to the $i$-th cluster center of $\Omega_{1}$ is recolored according to the subsequent interpolation-based equation,

$$
\boldsymbol{p}_{q_{1} q_{2}, \text { rec }}=\boldsymbol{r}_{i, r e c}+\left(\boldsymbol{p}_{i j}-\boldsymbol{r}_{i}\right)
$$

where $q_{1} \in\left\{1,2, \ldots, M_{1}\right\}, q_{2} \in\left\{1,2, \ldots, M_{2}\right\}, i=1,2, \ldots, n_{1}$. 
Thus, the above approach finally recolors all image pixels that are confused by the dichromat. Algorithm 1 illustrates the pseudo-code of the proposed image recoloring method.

\subsection{Algorithm 1}

Input: Image of size $M_{1} \times M_{2}$; Select values for the parameters $\lambda, \gamma$ and $\beta$; Select the parameters $D_{\text {particles }}, F r, C r$, and $t_{\max }$ for the implementation of the differential evolution algorithm (DE); For protanopia select a fixed value for $\alpha \in$ $[-0.5,0]$ and $\beta=1$ and for deuteranopia case select $\alpha=1$ and $\beta \in[-0.5,0]$.

Output: Recolored image.

\subsubsection{Step 1}

Apply the hybrid clustering to obtain a partition of the image's colors into $n$ clusters with centers $V=\left\{\boldsymbol{v}_{1}, \boldsymbol{v}_{2}, \ldots\right.$, $\left.v_{n}\right\}$, where $n$ is given in eq. (13).

\subsubsection{Step 2}

- Obtain the dichromatic simulation of the elements of $V$, denoted as $f_{D}\left(\boldsymbol{v}_{i}\right), i=1,2, \ldots, n$.

- Apply the eq. (14) to identify the confusing colors of $V$; divide $V$ into the set $\Omega_{1}=\left\{\boldsymbol{r}_{1}, \boldsymbol{r}_{2}, \ldots, \boldsymbol{r}_{n_{1}}\right\}$ that contains all confusing colors of $V$ and $\Omega_{2}=\left\{\boldsymbol{o}_{1}, \boldsymbol{o}_{2}, \ldots, \boldsymbol{o}_{n_{2}}\right\}$ that contains the intact colors of $V$, where $n_{1}+n_{2}=n$.

- Use the eqs (15) and (16) to calculate the dichromatic simulations for the elements of $\Omega_{1}$ and $\Omega_{2}$ as $\boldsymbol{r}_{i, D}=f_{D}\left(\boldsymbol{v}_{i}\right), i$ $=1,2, \ldots, n_{1}$ and $\boldsymbol{o}_{j, D}=f_{D}\left(\boldsymbol{o}_{j}\right), j=1,2, \ldots, n_{2}$.

\subsubsection{Step 3}

- For $i=1: n_{1}$

- Apply the eq. (18) to estimate the recoloring of the element $\boldsymbol{r}_{i} \in \Omega_{\mathrm{c}}$, denoted as $\boldsymbol{r}_{i, \text { rec }}$, which is a function of the parameters $\mu_{i, 21}, \mu_{i, 31}$ (for protanopia), and $\mu_{i, 12}, \mu_{i, 32}$ (for deuteranopia) of the matrix $\boldsymbol{A}_{i}$ given in eq. (19).

- Employ the eq. (20) to obtain the dichromatic simulation of $\boldsymbol{r}_{i, r e c}$, denoted as $\boldsymbol{r}_{i, r e c, D}$.

\section{EndFor}

- Formulate the objective function $E$ in eq. (21).

- Run the DE algorithm for $t_{\max }$ iterations to minimize $E$ with respect to parameters $\mu_{i, 21}, \mu_{i, 31}$ for the case of protanopia and $\mu_{i, 12}, \mu_{i, 32}$ for the case of deuteranopia.

\subsubsection{Step 4}

Apply the eq. (25) to recolor all confusing colors of the original image. This step yields the recolored image.

Next, the computational complexity of the method is determined in terms of the distance calculations per iteration. Since Step 2 and Step 4 are one pass through the data, it is obvious that the most dominant effects are coming from Step 1 and Step 3. Regarding Step 1, the objective function of hybrid clustering is presented in eq. (4). Given that the number of image's colors is $M_{1} M_{2}$ and the number of clusters is $n$, we can easily verify that the number of distance calculations per iteration is equal to $M_{1} M_{2} n+M_{1} M_{2} n(1+n)$. But $M_{1} M_{2} n+M_{1} M_{2} n(1+n)=2 M_{1} M_{2} n+M_{1} M_{2} n^{2}<3 M_{1} M_{2} n^{2}$. Thus, the complexity of the hybrid clustering applied in our case is $O\left(M_{1} M_{2} n^{2}\right)$.

Regarding Step 3, the DE employs $D_{\text {particles }}$ individuals (i.e., particles), each of which calculates the objective function in eq. (21) during each iteration. Thus, the number of distance calculations performed in (21) by DE is equal to $D_{\text {particles }}\left(3 n_{1} n_{2}+3 n_{1}^{2}+n_{1}\right)$. But $3 n_{1} n_{2}+3 n_{1}^{2}+n_{1}<6\left(n_{1} n_{2}+n_{1}^{2}\right)$ and therefore the computational complexity for Step 3 is $O\left(D_{\text {particles }}\left(6\left(n_{1} n_{2}+n_{1}^{2}\right)\right)\right)$.

In total, we obtain $O\left(M_{1} M_{2} n^{2}+D_{\text {particles }}\left(6\left(n_{1} n_{2}+n_{1}^{2}\right)\right)\right)$. Given that $6\left(n_{1} n_{2}+n_{1}^{2}\right)<12 n^{2}$, it follows $O\left(M_{1} M_{2} n^{2}+\right.$ 
$\left.12 D_{\text {particles }} n^{2}\right)=O\left(\left(M_{1} M_{2}+12 D_{\text {particles }}\right) n^{2}\right) \sim O\left(n^{2}\right)$.

Thus, the complexity of the overall methodology is $O\left(n^{2}\right)$.

Remark 1. For the clarity of our presentation, we point out the differences between the present contribution and our previous work in [6]. There exist three major differences. First, in [6] the image colors are grouped using the standard fuzzy $k$-means. However, this approach obtains high computational costs, especially when considering largesized images. In addition, the implementation of the fuzzy $k$-means is based on assigning each image color to the group that appears the maximum membership degree, although that degree could be much smaller than 1 . Such an interpretation of the fuzzy $k$-means may assign an image color that is perceived correctly by the color blind to a cluster that must be recolored. As a result, that image color will be modified, although it is not necessary. On the contrary, the current approach utilizes a clustering methodology able to resolve the above problem by producing clusters that possess crisp and fuzzy areas. It has been shown previously that, as the learning process proceeds, the crisp areas increase their size. Thus, a significant amount of image colors clearly belongs to certain clusters and the decision to recolor them or not is clear, also. On the other hand, image colors that belong to the fuzzy area of a cluster are distant from the corresponding representative color. Thus, even in the worst-case scenario where an image color is misclassified, the respective effect will not have a strong impact on the final recoloring result as far as the naturalness and contrast enhancement criteria are concerned. Second, in [6], the recoloring of the cluster centers is conducted through a process that involves the confusion lines in the XYZ color space. Specifically, if a confusion line contains more than one cluster center, all but one center are relocated to different confusion lines so that they can be distinguished by the color-blind. The final recoloring is obtained by minimizing an objective function with respect to the luminance coordinate in the $\mathrm{XYZ}$ color space. In the present contribution, the recoloring is accomplished in terms of a daltonization process in the RGB space and involves a matrix-based transformation to accurately relate the original and the respective dichromacy simulated colors. Then, an objective function is minimized with respect to the parameters of the matrix used to perform the above transformation. Note that, although the objective functions in the two cases are the same, the optimization procedures are quite different since they are minimized with respect to different parameters and objectives. Third, in [6], the image colors assigned to a cluster center that must be modified, are recolored using a color transfer approach. On the other hand, in the present contribution, the recoloring process of those image colors is carried out in terms of an interpolation-based mechanism. In summary, apart from the objective function form, the two methods are totally different.

\section{Simulation experiments}

In this section, the proposed methodology is tested and evaluated in terms of a comparative analysis considering three more related approaches. The first one, called Method 1, was developed by Huang et al. in [29], the second one, called Method 2, was proposed by Wong and Bishop in [9], and the third one was developed by Chatzistamatis et al. in [8], called Method 3. The parameter values for the proposed algorithm are selected as: $\lambda=0.5, \gamma=0.5, \delta=30, D_{\text {particles }}=$ $20, F r=0.8, C r=0.6$, and $t_{\max }=200$. Finally, considering the matrices in eq. (19), for the protanopia case we selected $\alpha$ $=-0.5$ and $\beta=1$, while for the deuteranopia case, $\alpha=1$ and $\beta=-0.5$. For the other three methods the parameter settings were the same as in the respective references.

Two performance indices are used to conduct the experimental evaluation and comparison. The first index is the naturalness index developed in [8], which is calculated as follows,

$$
J_{n a t}=\frac{1}{M_{1} M_{2}} \sum_{q_{1}}^{M_{1}} \sum_{q_{2}}^{M_{2}}\left\|\boldsymbol{p}_{q_{1} q_{2}}-\boldsymbol{p}_{q_{1} q_{2}, r e c}\right\|
$$

As the value of the index $J_{n a t}$ decreases the recolored image appears to be more similar to the original obtaining natural color distribution between different image areas $[6,8,11]$.

The second index is the feature similarity index $(F S I M c)$ developed in [38]. For larger values of that index the chrominance of the recolored image gets closer to the respective chrominance of the original image $[6,38]$. The 
analytical derivation of the index FSIMc is given in [38].

In what follows, three types of experimental studies are discussed namely, quantitative, qualitative, and subjective evaluation, which are presented within the subsequent sections.

\subsection{Quantitative evaluation}

In this section, the four methods are compared in terms of rigorous statistical analysis using the above-mentioned indices. To evaluate the results, we used 45 digitized art paintings, influenced by Naturalism and Cubism, taken from the WikiArt [40]. The data set includes 45 art paintings. Table 1 provides the details of the tested paintings.

Table 1. The tested paintings taken from the WikiArt

\begin{tabular}{|c|c|c|c|c|c|}
\hline \# & Painting & Painter & $\#$ & Painting & Painter \\
\hline 1 & A Bowl of Plums & Chardin & 24 & Lands & Lazlo Nagy \\
\hline 2 & Swans & Kevin Sloan & 25 & Les pr Sences & Leopold Survage \\
\hline 3 & Bird Painting & Timothy Easton & 26 & Woman and Bird & Leopold Survage \\
\hline 4 & Bedroom Oil Canvas & Vincent Van Gogh & 27 & The Large Fly & Louis Marcoussis \\
\hline 5 & Still Life with Fruit Bowl & Corneliu Michailescu & 28 & Violin Bottle Flowers & Louis Marcoussis \\
\hline 6 & Corner of the Garden & Dorrit Black & 29 & Flor de Pascua & Marianne North \\
\hline 7 & Dutch Houses & Dorrit Black & 30 & Homage to Friends & Marie Vorobieff \\
\hline 8 & Elizabeth Street Sydney & Dorrit Black & 31 & Composition 1 & Patrick Bruce \\
\hline 9 & Abstract Composition & Erich Buchholz & 32 & House in the Field & Pablo Picasso \\
\hline 10 & Cows & Vincent Van Gogh & 33 & $\begin{array}{l}\text { Gestufte Quadratreihe um } \\
\text { ein Komplementrzentum }\end{array}$ & Richard Lohse \\
\hline 11 & A Vase from Vieux & Gustave Buchet & 34 & The Blue Vase & Paul Cezanne \\
\hline 12 & Nature Morte avec des Livres & Gustave Buchet & 35 & Still Life & Vilhelm Lundstorm \\
\hline 13 & Interior with Guitar & Henri Fauconnie & 36 & The Chess Game & Anguissola Sofonisba \\
\hline 14 & The Signal & Henri Fauconnie & 37 & A Walk with Grandpa & Augustus Edwin Mulready \\
\hline 15 & Ducks & Jessie Arms Botke & 38 & Knight Death and Girl & Hans Baldung \\
\hline 16 & Puerto de Barcelona & Joaquin Garcia & 39 & Two Girls & Harlamov \\
\hline 17 & Jaro & Josef Capek & 40 & The Contrast & Henry Hunt \\
\hline 18 & Bananas & Juan Gris & 41 & St. Geroge and the Dragon & Raphael \\
\hline 19 & Fruit with Bowl & Juan Gris & 42 & Birds in a Landscape & Tobias Stanover \\
\hline 20 & Interiour & Kmetty Janos & 43 & Paesaggio con Fiume & Tessari Vittorio \\
\hline 21 & Still Life & Kmetty Janos & 44 & St. Geroge and the Dragon & Uccello \\
\hline 22 & Szentendre & Kmetty Janos & 45 & Stedelijk & Vincent Van Gogh \\
\hline 23 & Constructivist Composition & Lajos Kassak & & & \\
\hline
\end{tabular}


Regarding the two indices, the descriptive statistics for protanopia and deuteranopia are depicted in Tables 2 and 3 , respectively. Figures 2 and 3 illustrate the corresponding box-plots. The above results reinforce the hypothesis that a statistically significant difference exists between our method and the other three methods. To examine the hypothesis, we employ non-parametric statistical inference. Pairwise comparisons between our proposed method and the other three methods were conducted using the Wilcoxon signed rank-sum test. The null hypothesis is that the median of the population of differences between the paired data is zero, at overall 0.05 significance level.

Table 2. Descriptive statistics of the $J_{\text {nat }}$ and FSIMc indices for the protanopia case

\begin{tabular}{lccccccc}
\hline & Minimum & Q1 & Median $(\mathrm{Q} 2)$ & Q3 & Maximum & Mean & Std. Deviation \\
\hline Proposed & 0.4550 & 1.6531 & 2.8494 & 5.4249 & 12.3320 & 3.7378 & 2.7851 \\
Method 1 & 5.1288 & 7.2252 & 10.5108 & 12.4151 & 16.6370 & 10.1326 & 3.2743 \\
Method 2 & 2.6378 & 6.5413 & 8.4510 & 10.2132 & 13.2070 & 8.4276 & 2.4935 \\
Method 3 & 1.2985 & 4.6358 & 7.5125 & 10.3160 & 15.7502 & 7.3574 & 3.4996 \\
& & & & & & & \\
Proposed & 0.9274 & 0.9720 & 0.9793 & 0.9864 & 0.9942 & 0.9754 & 0.0164 \\
Method 1 & 0.9065 & 0.9566 & 0.9726 & 0.9853 & 0.9957 & 0.9677 & 0.0212 \\
Method 2 & 0.8786 & 0.9304 & 0.9462 & 0.9557 & 0.9682 & 0.9393 & 0.0214 \\
Method 3 & 0.9246 & 0.9614 & 0.9751 & 0.9817 & 0.9932 & 0.9712 & 0.0159 \\
\hline
\end{tabular}

Table 3. Descriptive statistics of the $J_{\text {nat }}$ and FSIMc indices for the deuteranopia case

\begin{tabular}{|c|c|c|c|c|c|c|c|}
\hline & & & & $J_{\text {nat }}$ & & & \\
\hline Proposed & 0.4703 & 1.9973 & 3.5246 & 5.1560 & 9.3879 & 3.7589 & 2.3010 \\
\hline Method 1 & 0.2135 & 3.4595 & 7.9796 & 11.0165 & 17.1382 & 7.5208 & 4.4836 \\
\hline Method 2 & 2.6668 & 6.2501 & 8.3845 & 10.2662 & 12.9797 & 8.3013 & 2.5467 \\
\hline \multirow[t]{2}{*}{ Method 3} & 0.7875 & 3.7469 & 6.2029 & 8.0210 & 12.4981 & 6.1304 & 2.8693 \\
\hline & & & & FSIMc & & & \\
\hline Method 2 & 0.8838 & 0.9302 & 0.9468 & 0.9568 & 0.9694 & 0.9397 & 0.0219 \\
\hline Method 3 & 0.8373 & 0.9425 & 0.9590 & 0.9679 & 0.9837 & 0.9521 & 0.0258 \\
\hline
\end{tabular}




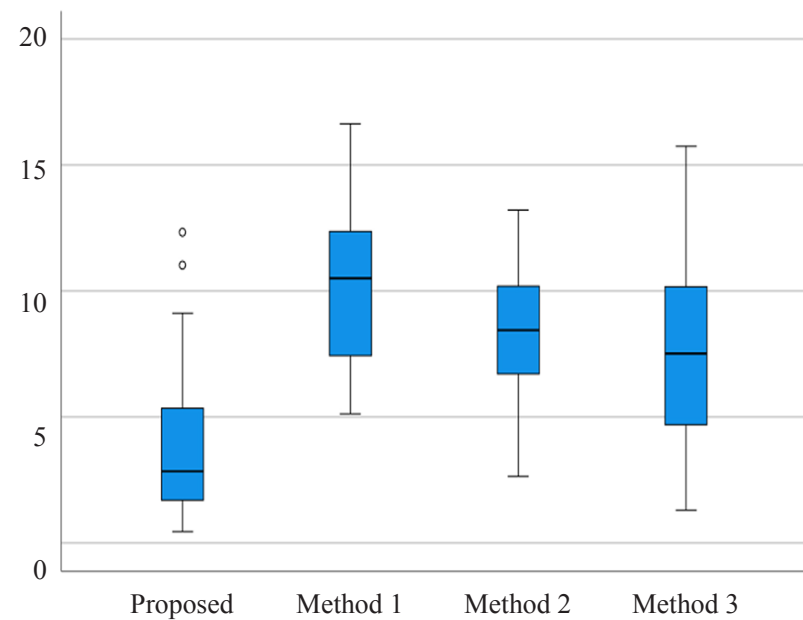

(a)

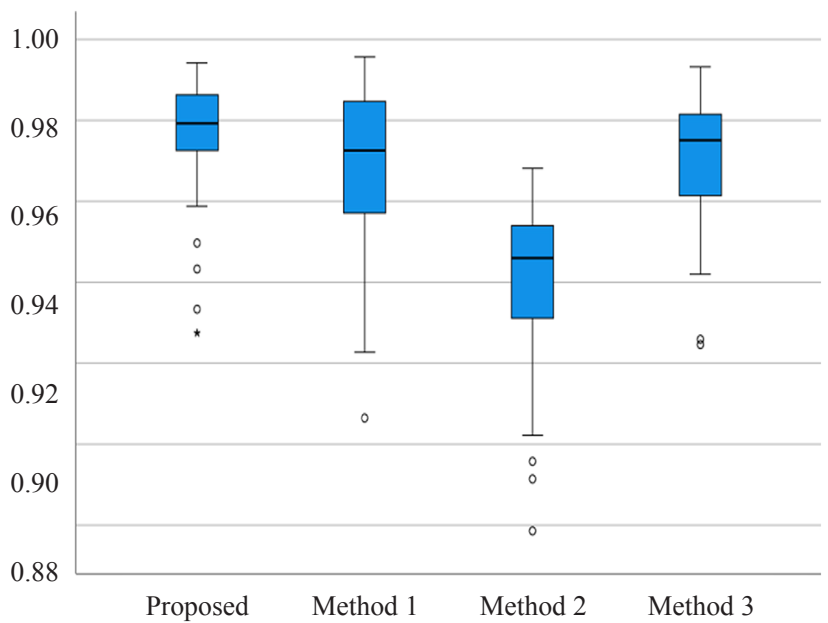

(b)

Figure 2. Boxplots for protanopia case: (a) the $J_{\text {nat }}$ index, (b) FSIMc index

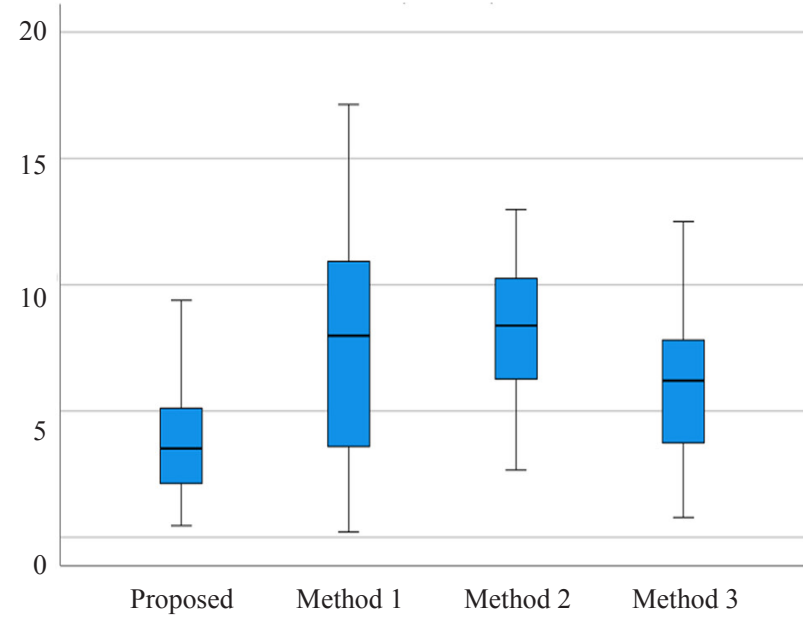

(a)

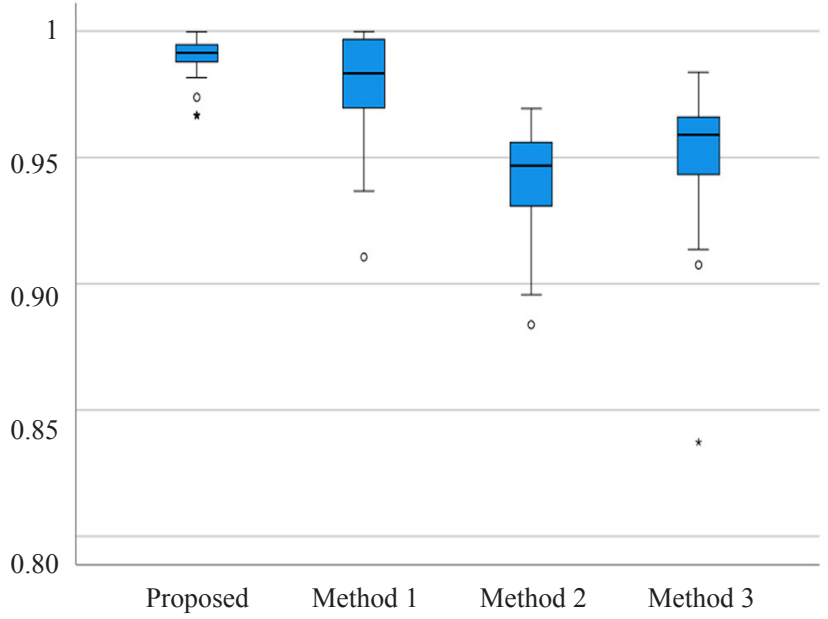

(b)

Figure 3. Boxplots for deuteranopia case: (a) the $J_{\text {nat }}$ index, (b) FSIMc index

Table 4 and Table 6 summarize the $J_{\text {nat }}$ results for protanopia. According to Table 6 , we rejected the null hypothesis in favor of the alternative at significantly less than 0.001 . The positive ranks at Table 4 (method-proposed method) state that the $J_{n a t}$ obtained by our proposed method is significantly lower when compared to each one of the other methods.

Table 5 and Table 6 summarize the FSIMc results for protanopia. The negative ranks at Table 5 (method-proposed method) declare that the proposed method FSIMc index is significantly greater than each one of the other methods. The null hypothesis is rejected (see Table 6) thus the proposed method is superior to the other three compared methods.

Table 7 and Table 9 summarize the $J_{\text {nat }}$ results for the deuteranopia case. According to Table 9, we reject the null hypothesis in favor of the alternative at significance less than 0.001 . The positive ranks in Table 7 (compared methodproposed method) state that the $J_{n a t}$ of the compared method index is significantly greater than the proposed method. We conclude that our method is superior to the other three, preserving naturalness of the recolored image. 
Table 4. Wilcoxon mean rank and sum of ranks of the $J_{\text {nat }}$ index for protanopia

\begin{tabular}{|c|c|c|c|}
\hline & No of paintings & Mean Rank & Sum of Ranks \\
\hline \multicolumn{4}{|c|}{ Method 1-Proposed } \\
\hline Negative Ranks & 0 & 0.00 & 0.00 \\
\hline Positive Ranks & 45 & 23.00 & 1035.00 \\
\hline Ties & 0 & & \\
\hline \multicolumn{4}{|c|}{ Method 2-Proposed } \\
\hline Negative Ranks & 4 & 9.50 & 38.00 \\
\hline Positive Ranks & 41 & 24.32 & 997.00 \\
\hline Ties & 0 & & \\
\hline \multicolumn{4}{|c|}{ Method 3-Proposed } \\
\hline Negative Ranks & 0 & 0.00 & 0.00 \\
\hline Positive Ranks & 45 & 23.00 & 1035.00 \\
\hline Ties & 0 & - & - \\
\hline
\end{tabular}

Table 5. Wilcoxon mean rank and sum of ranks of the FSIMc index for protanopia

\begin{tabular}{|c|c|c|c|}
\hline & No of paintings & Mean Rank & Sum of Ranks \\
\hline \multicolumn{4}{|c|}{ Method 1-Proposed } \\
\hline Negative Ranks & 32 & 23.53 & 753.00 \\
\hline Positive Ranks & 13 & 21.69 & 282.00 \\
\hline Ties & 0 & - & - \\
\hline \multicolumn{4}{|c|}{ Method 2-Proposed } \\
\hline Negative Ranks & 45 & 23.00 & 1035.00 \\
\hline Positive Ranks & 0 & 0.00 & 0.00 \\
\hline Ties & 0 & - & - \\
\hline \multicolumn{4}{|c|}{ Method 3-Proposed } \\
\hline Negative Ranks & 30 & 24.63 & 739.00 \\
\hline Positive Ranks & 14 & 17.93 & 251.00 \\
\hline Ties & 1 & - & - \\
\hline
\end{tabular}


Table 6. Wilcoxon test statistic Z-value and asymptotic significance of $J_{\text {nat }}$ and FSIMc indices for protanopia

\begin{tabular}{|c|c|c|c|}
\hline & Method 1-Proposed & Method 2-Proposed & Method 3-Proposed \\
\hline \multicolumn{4}{|c|}{$J_{\text {nat }}$} \\
\hline Z & -5.841 & -5.412 & -5.841 \\
\hline Asymp. Significance & $<0.001$ & $<0.001$ & $<0.001$ \\
\hline \multicolumn{4}{|c|}{ FSIMc } \\
\hline & Method 1-Proposed & Method 2-Proposed & Method 3-Proposed \\
\hline Z & -2.658 & -5.841 & -2.848 \\
\hline Asymp. Significance & 0.008 & $<0.001$ & 0.004 \\
\hline
\end{tabular}

Table 7. Wilcoxon test statistic mean rank and sum of ranks of the $J_{\text {nat }}$ index for deuteranopia

\begin{tabular}{|c|c|c|c|}
\hline & No of paintings & Mean Rank & Sum of Ranks \\
\hline \multicolumn{4}{|c|}{ Method 1-Proposed } \\
\hline Negative Ranks & 12 & 12.08 & 145.00 \\
\hline Positive Ranks & 33 & 26.97 & 890.00 \\
\hline Ties & 0 & - & - \\
\hline \multicolumn{4}{|c|}{ Method 2-Proposed } \\
\hline Negative Ranks & 3 & 9.33 & 28.00 \\
\hline Positive Ranks & 42 & 23.98 & 23.98 \\
\hline Ties & 0 & - & - \\
\hline \multicolumn{4}{|c|}{ Method 3-Proposed } \\
\hline Negative Ranks & 1 & 1.00 & 1.00 \\
\hline Positive Ranks & 44 & 23.50 & 1034.00 \\
\hline Ties & 0 & - & - \\
\hline
\end{tabular}

Table 8 and Table 9 summarize the FSIMc Wilcoxon test for deuteranopia. The negative ranks at Table 8 (compared method-proposed method) declare that the proposed method FSIMc index is significantly greater than the compared methods. The null hypothesis is rejected (Table 9) thus proposed method is superior to the other three compared method.

In summary, there was a statistically significant difference between the proposed method the other three methods. Wilcoxon test revealed that our proposed method outperformed the other three in terms of preserving the naturalness of the recolored image for the two dichromacy cases. The above statement is further examined in the qualitative evaluation. Furthermore, our proposed method was superior in terms of the FSIMc index for both protanopia and deuteranopia. 
Table 8. Wilcoxon test statistic mean rank and sum of ranks of the FSIMc index for deuteranopia

\begin{tabular}{|c|c|c|c|}
\hline & No of paintings & Mean Rank & Sum of Ranks \\
\hline \multicolumn{4}{|c|}{ Method 1-Proposed } \\
\hline Negative Ranks & 31 & 24.97 & 774.00 \\
\hline Positive Ranks & 14 & 18.64 & 261.00 \\
\hline Ties & 0 & - & - \\
\hline \multicolumn{4}{|c|}{ Method 2-Proposed } \\
\hline Negative Ranks & 45 & 23.00 & 1035.00 \\
\hline Positive Ranks & 0 & 0.00 & 0.00 \\
\hline Ties & 0 & - & - \\
\hline \multicolumn{4}{|c|}{ Method 3-Proposed } \\
\hline Negative Ranks & 45 & 23.00 & 1035.00 \\
\hline Positive Ranks & 0 & 0.00 & 0.00 \\
\hline Ties & 0 & - & - \\
\hline
\end{tabular}

Table 9. Wilcoxon test statistic Z-value and asymptotic significance of $J_{\text {nat }}$ and FSIMc indices for deuteranopia

\begin{tabular}{|c|c|c|c|}
\hline & Method 1-Proposed & Method 2-Proposed & Method 3-Proposed \\
\hline \multicolumn{4}{|c|}{$J_{\text {nat }}$} \\
\hline Z & -4.205 & -5.525 & -5.830 \\
\hline \multirow[t]{4}{*}{ Asymp. Significance } & $<0.001$ & $<0.001$ & $<0.001$ \\
\hline & & - & - \\
\hline & \multicolumn{2}{|c|}{ FSIMc } & \\
\hline & Method 1-Proposed & Method 2-Proposed & Method 3-Proposed \\
\hline Z & -2.895 & -5.841 & -5.841 \\
\hline Asymp. Significance & 0.004 & $<0.001$ & $<0.001$ \\
\hline
\end{tabular}

\subsection{Qualitative evaluation}

In this section, the algorithm is tested and evaluated using the six paintings depicted in Figure 4. The paintings were from the WikiArt database [40]. 


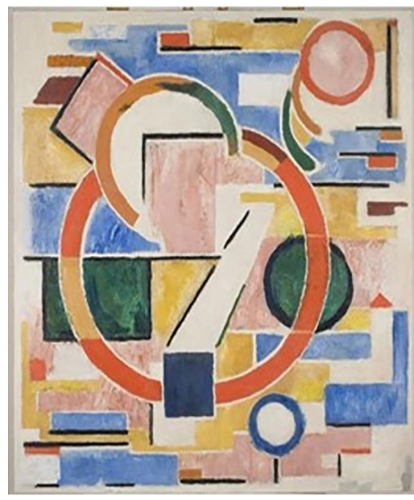

(a)

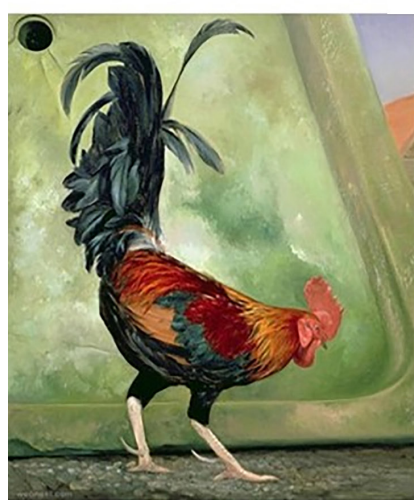

(d)

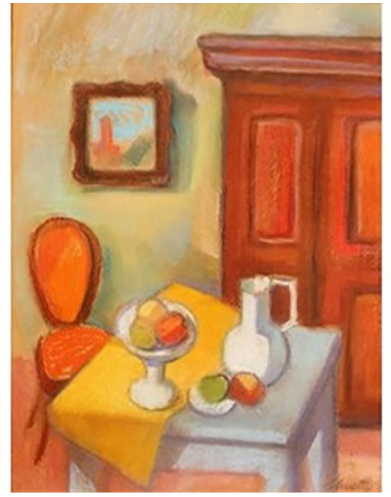

(b)

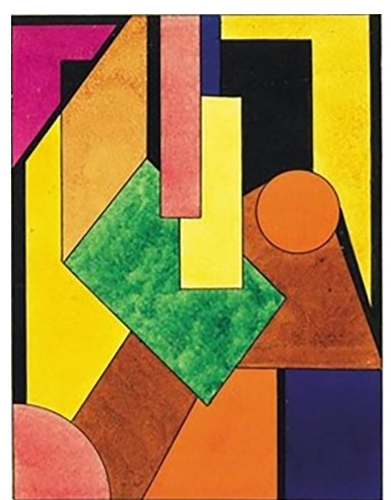

(e)

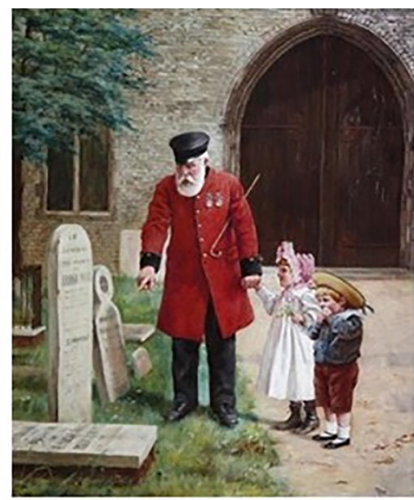

(c)

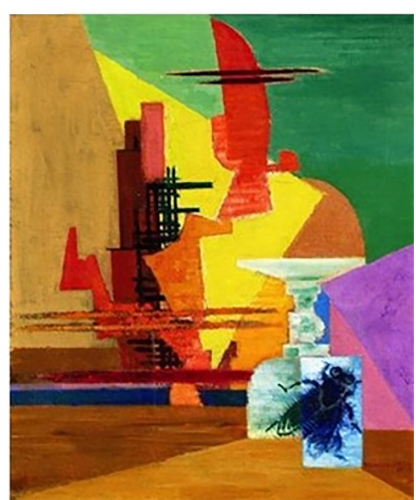

(f)

Figure 4. The tested art paintings: (a) Painting 1, (b) Painting 2, (c) Painting 3, (d) Painting 4, (e) Painting 5, (f) Painting 6

Table 10 and Table 11 depict the results obtained by the $J_{\text {nat }}$ index for the two dichromacy cases, while Table 12 and Table 13 illustrate the respective results for the FSIMc index. As far the $J_{\text {nat }}$ is concerned (see Tables 10 and 11), apart from few cases (i.e., painting 2 and painting 6), the proposed method significantly outperformed the other two methods. Similar results are extracted in Tables 12 and 13 that concern the FSIMc index.

Table 10. Results for the performance index $J_{\text {nat }}$ for protanopia

\begin{tabular}{ccccc}
\hline \multirow{2}{*}{ Painting } & \multicolumn{4}{c}{ Method } \\
\cline { 2 - 5 } & Proposed & Method 1 & Method 2 & Method 3 \\
\hline Painting 1 & 1.496 & 6.997 & 8.267 & 7.9221 \\
Painting 2 & 8.4988 & 5.9642 & 14.4606 & 12.7021 \\
Painting 3 & 1.2838 & 5.4204 & 7.5036 & 2.5504 \\
Painting 4 & 1.1616 & 13.6899 & 12.966 & 2.3714 \\
Painting 5 & 1.502 & 7.973 & 8.451 & 4.2324 \\
Painting 6 & 12.332 & 16.637 & 15.7503 & 8.8958 \\
\hline
\end{tabular}


Table 11. Results for the performance index $J_{n a t}$ for deuteranopia

\begin{tabular}{ccccc}
\hline \multirow{2}{*}{ Image } & \multicolumn{4}{c}{ Method } \\
\cline { 2 - 5 } & Proposed & Method 1 & Method 2 & Method 3 \\
\hline Painting 1 & 3.1701 & 5.9516 & 8.2676 & 7.0551 \\
Painting 2 & 8.5956 & 9.232 & 11.4727 & 5.3714 \\
Painting 3 & 3.7708 & 8.192 & 8.2297 & 6.2029 \\
Painting 4 & 1.0463 & 14.3282 & 12.9797 & 2.5011 \\
Painting 5 & 1.86 & 7.409 & 8.4247 & 3.7468 \\
Painting 6 & 9.3879 & 13.816 & 8.8842 & 12.4981 \\
\hline
\end{tabular}

Table 12. Results for the performance index FSIMc for protanopia

\begin{tabular}{ccccc}
\hline \multirow{2}{*}{ Image } & \multicolumn{4}{c}{ Method } \\
\cline { 2 - 5 } & Proposed & Method 1 & Method 2 & Method 3 \\
\hline Painting 1 & 0.973 & 0.988 & 0.954 & 0.9753 \\
Painting 2 & 0.975 & 0.9321 & 0.9022 & 0.9514 \\
Painting 3 & 0.9926 & 0.9847 & 0.9576 & 0.9871 \\
Painting 4 & 0.9863 & 0.9423 & 0.9479 & 0.9859 \\
Painting 5 & 0.981 & 0.978 & 0.946 & 0.9672 \\
Painting 6 & 0.9374 & 0.9227 & 0.8958 & 0.9259 \\
\hline
\end{tabular}

Table 13. Results for the performance index FSIMc for deuteranopia

\begin{tabular}{ccccc}
\hline \multirow{2}{*}{ Image } & \multicolumn{3}{c}{ Method } \\
\cline { 2 - 4 } & Proposed & Method 1 & Method 2 & Method 3 \\
\hline Painting 1 & 0.9882 & 0.9866 & 0.9538 & 0.9489 \\
Painting 2 & 0.9664 & 0.9712 & 0.9026 & 0.9185 \\
Painting 3 & 0.9914 & 0.9835 & 0.9534 & 0.9608 \\
Painting 4 & 0.9955 & 0.9543 & 0.9479 & 0.9698 \\
Painting 5 & 0.992 & 0.9834 & 0.9464 & 0.9553 \\
Painting 6 & 0.9738 & 0.943 & 0.8957 & 0.9075999 \\
\hline
\end{tabular}


In the next experiment we consider qualitative comparisons over the six paintings. Figures 5, 6, and 7 show the qualitative comparisons, regarding protanopia, for Paintings 1, 2, and 3, respectively. Relationally, Figures 8, 9, and 10 illustrate the qualitative results, regarding deuteranopia, for Paintings 4, 5, and 6, respectively.

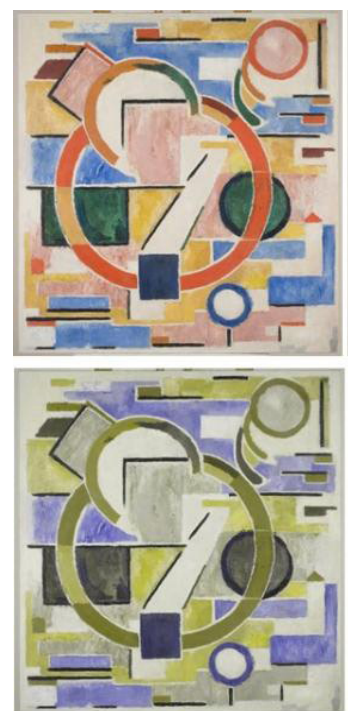

Original
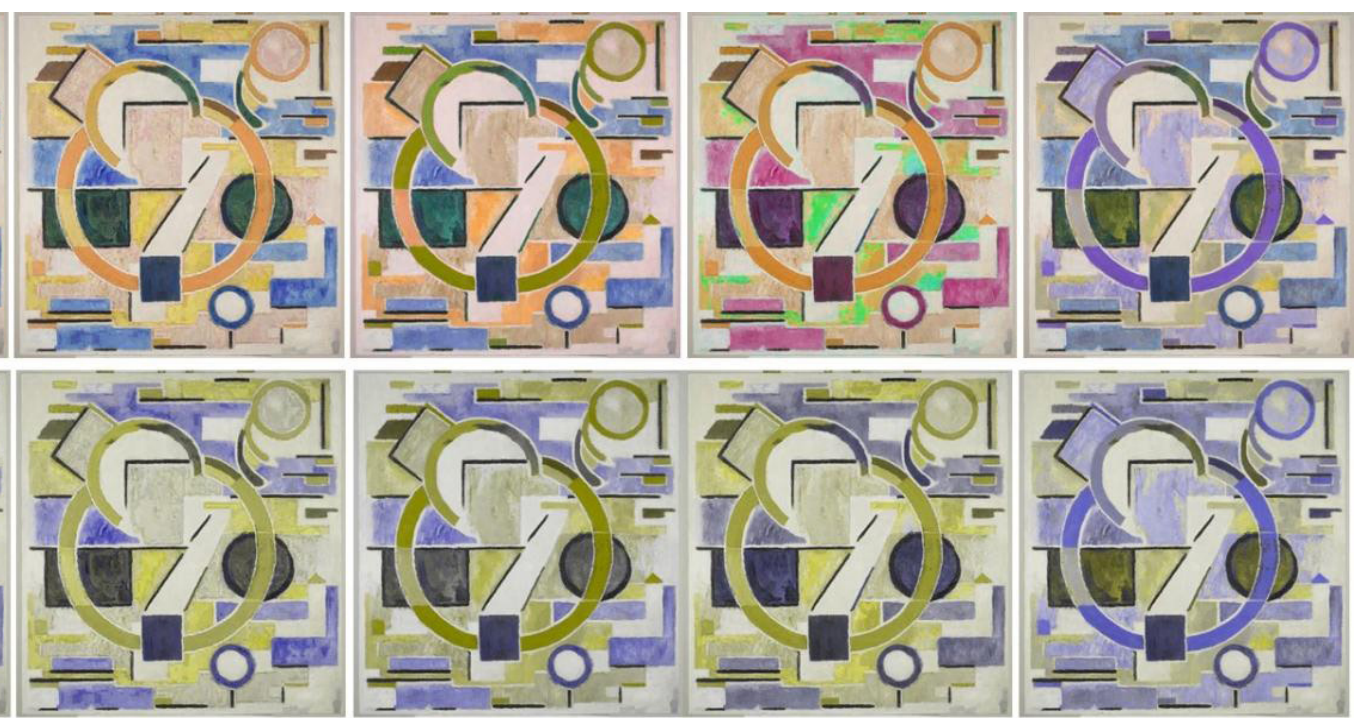

Proposed

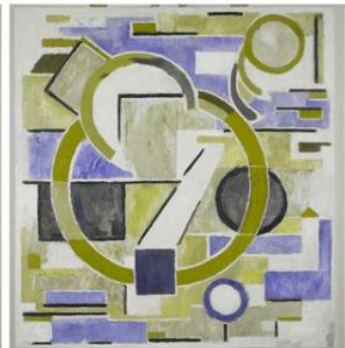

Method 1

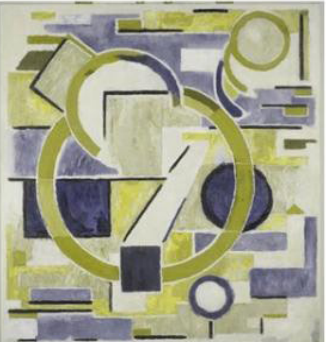

Method 2

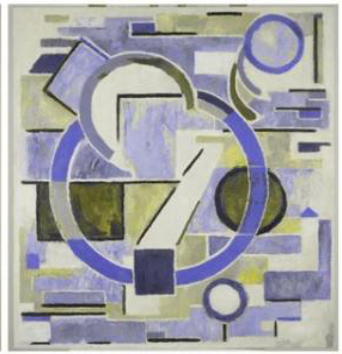

Method 3

Figure 5. Painting 1: Qualitative comparison for protanopia; The original and the recolored paintings are given in the first row, while their respective protanope simulations are given in the second row.
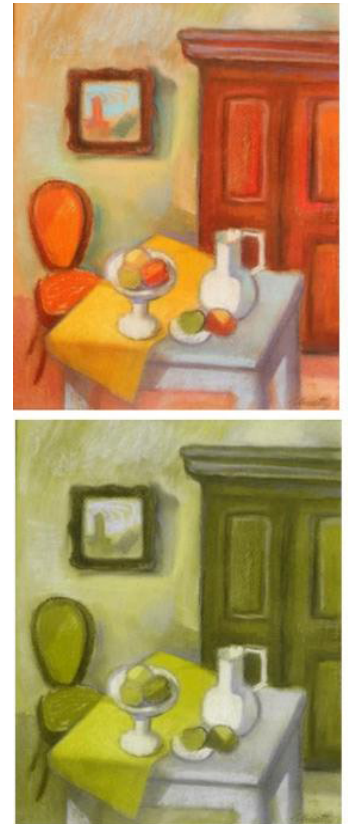

Original
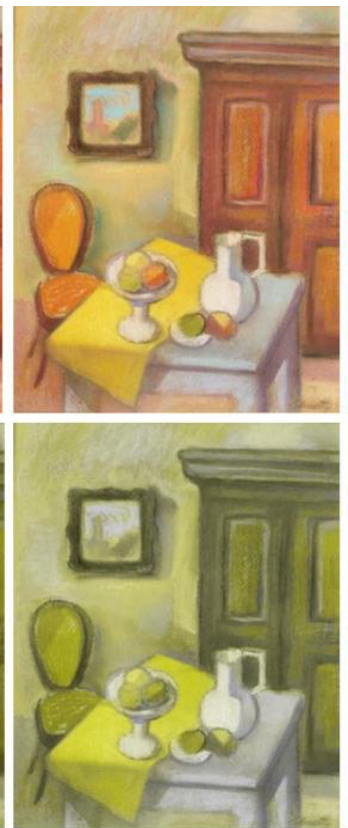

Proposed
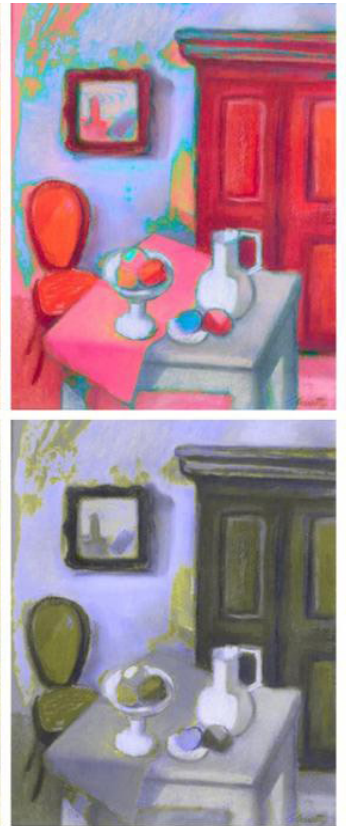

Method 1
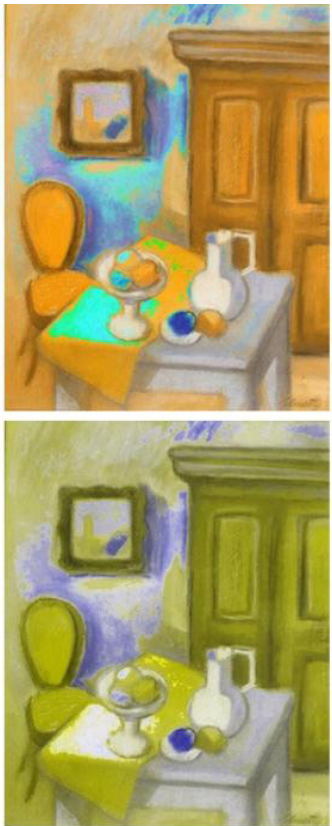

Method 2
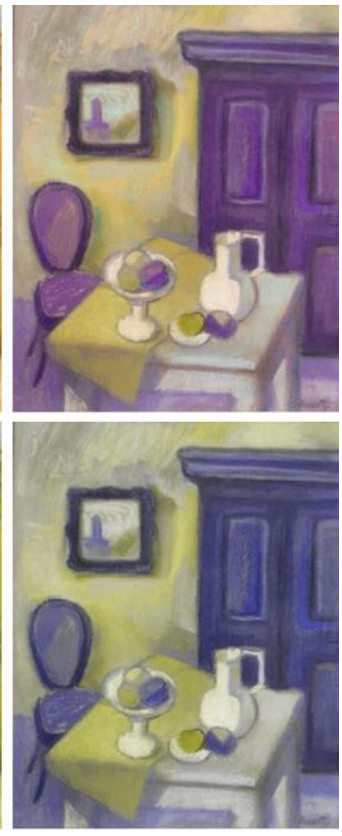

Method 3

Figure 6. Painting 2: Qualitative comparison for protanopia; The original and the recolored paintings are given in the first row, while their respective protanope simulations are given in the second row. 

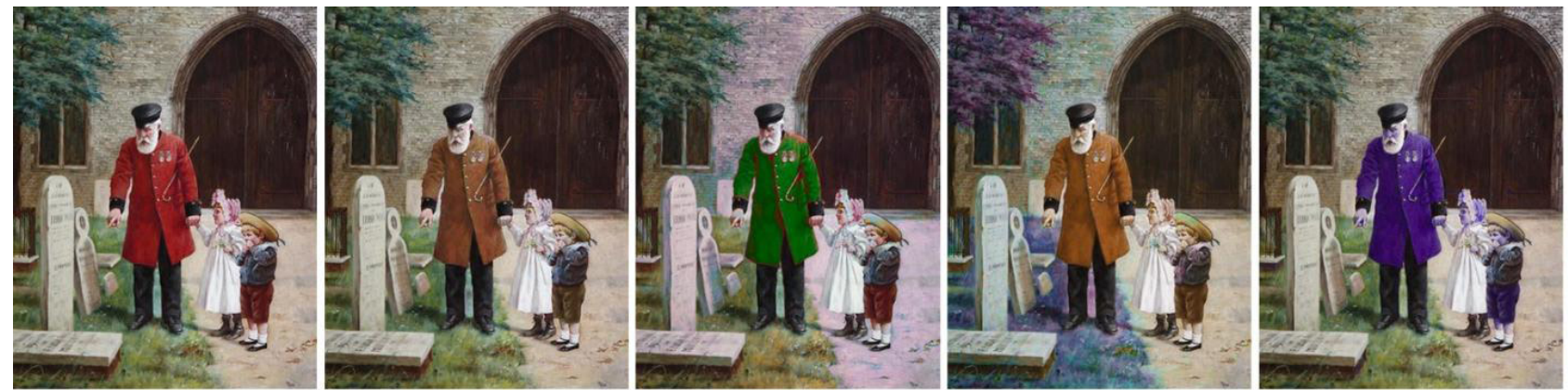

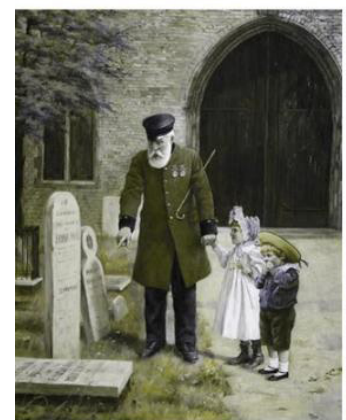

Original

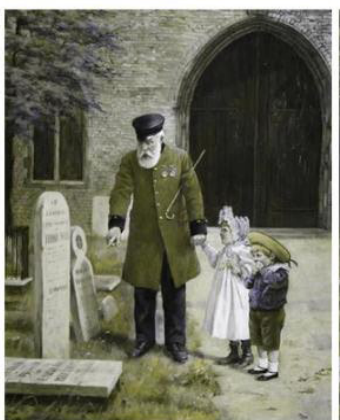

Proposed

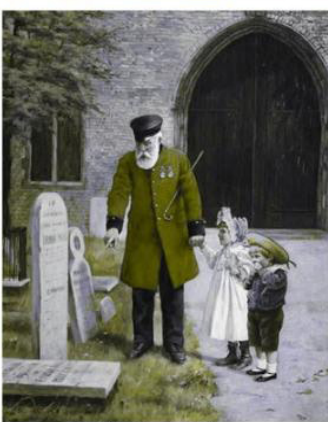

Method 1

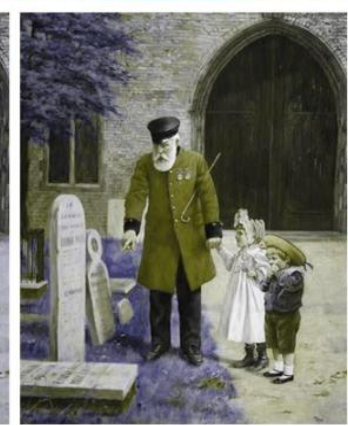

Method 2

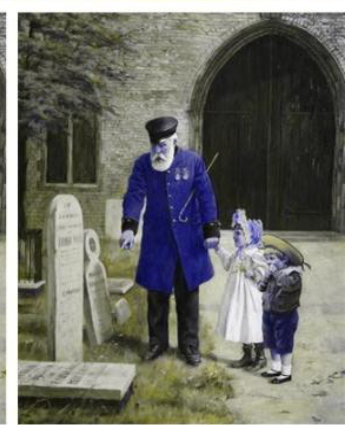

Method 3

Figure 7. Painting 3: Qualitative comparison for protanopia; The original and the recolored paintings are given in the first row, while their respective protanope simulations are given in the second row.
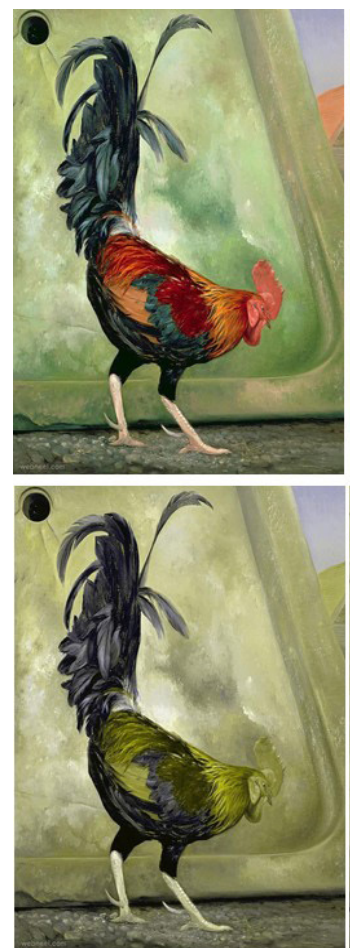

Original
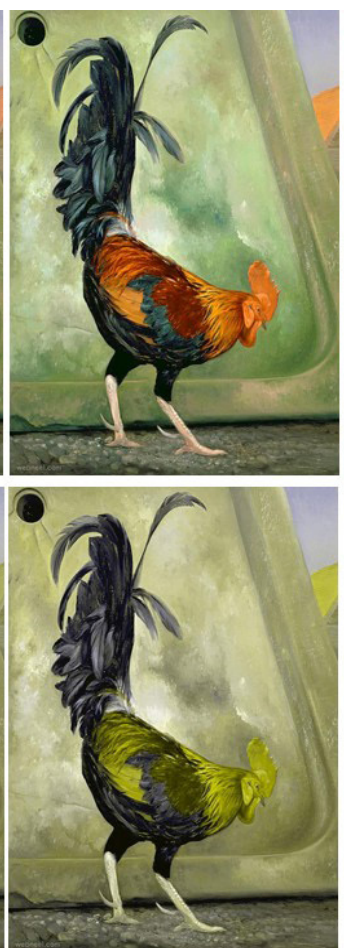

Proposed
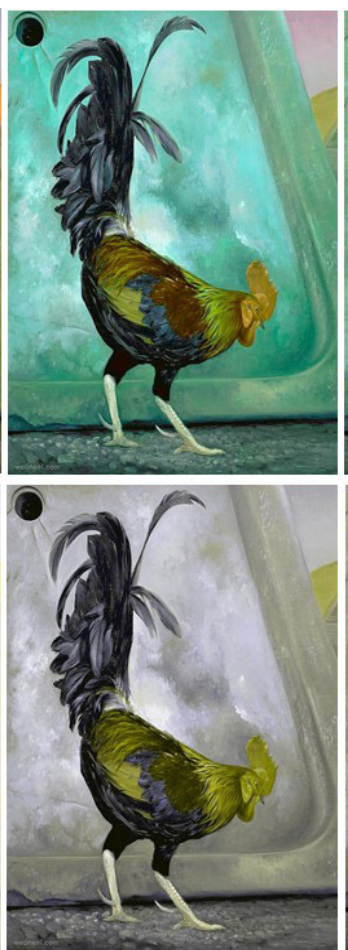

Method 1
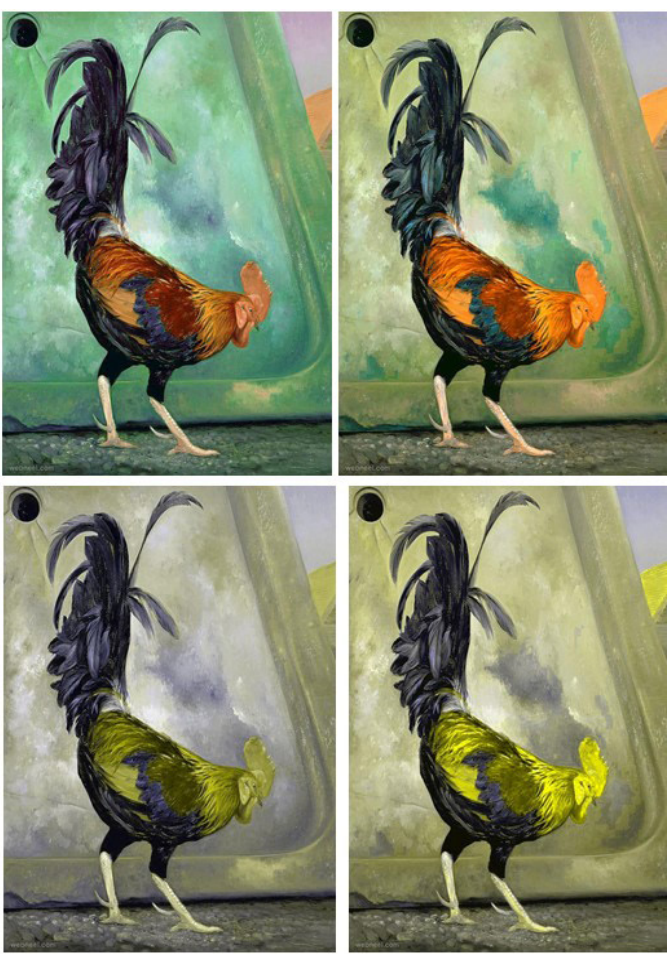

Method 2

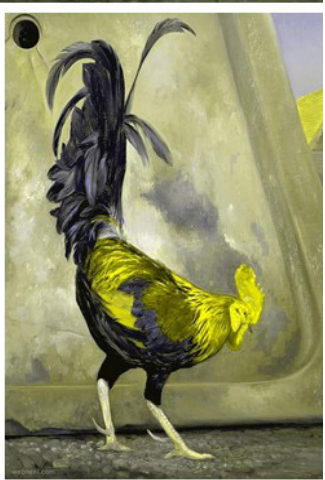

Method 3

Figure 8. Painting 4: Qualitative comparison for deuteranopia; The original and the recolored paintings are given in the first row, while their respective deuteranope simulations are given in the second row. 

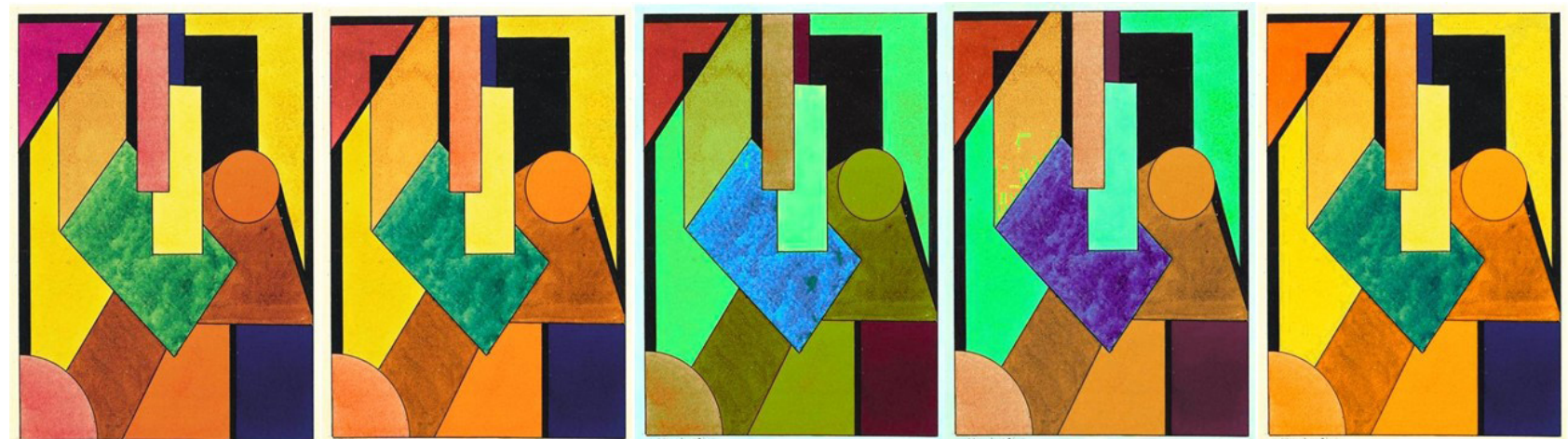

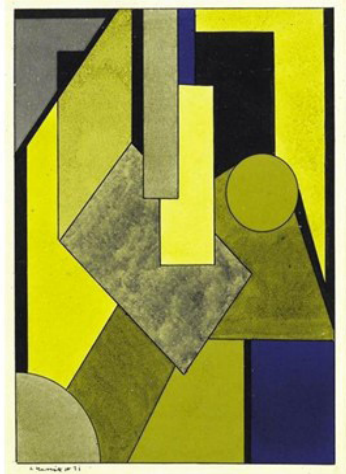

Original

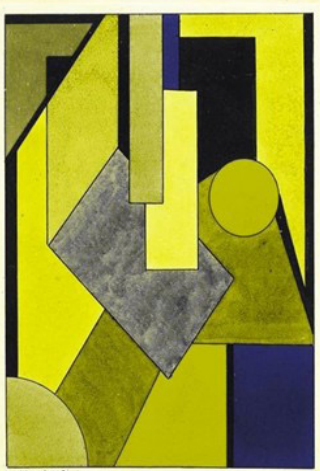

Proposed

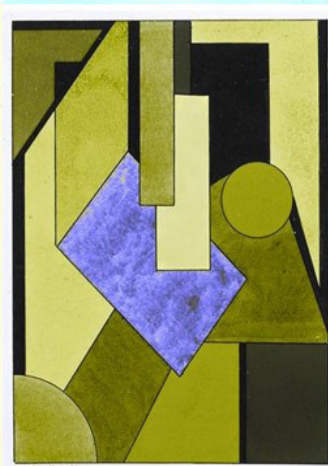

Method 1

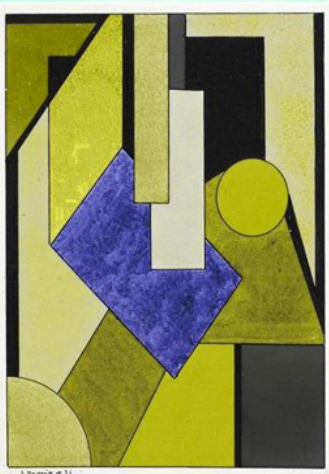

Method 2

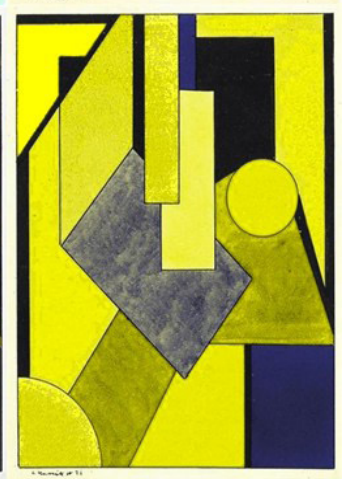

Method 3

Figure 9. Painting 5: Qualitative comparison for deuteranopia; The original and the recolored paintings are given in the first row, while their respective deuteranope simulations are given in the second row.
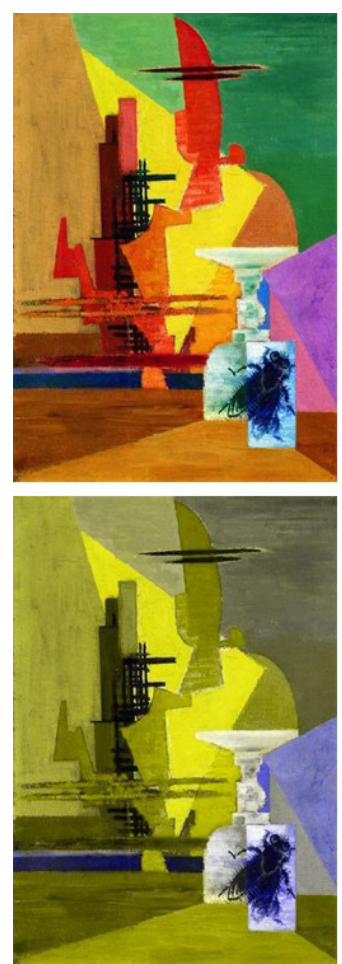

Original
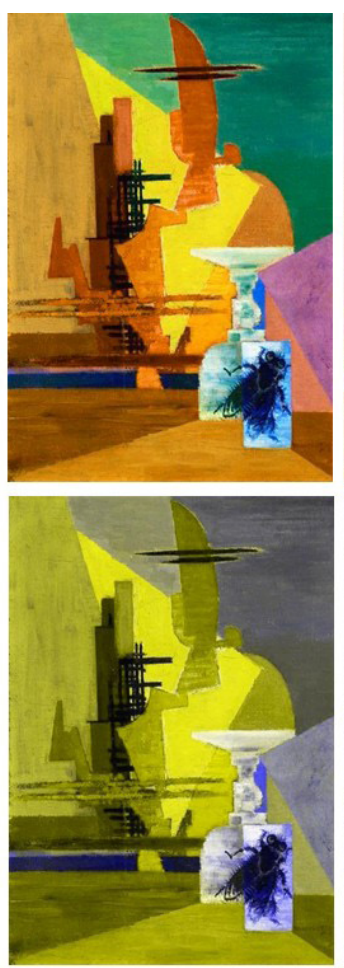

Proposed
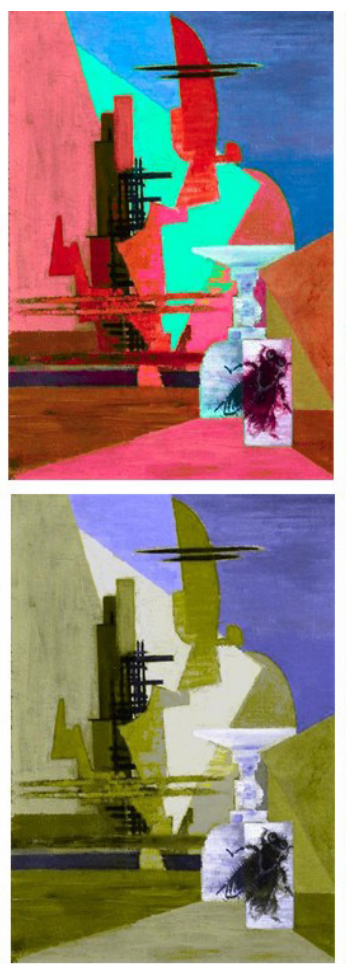

Method 1
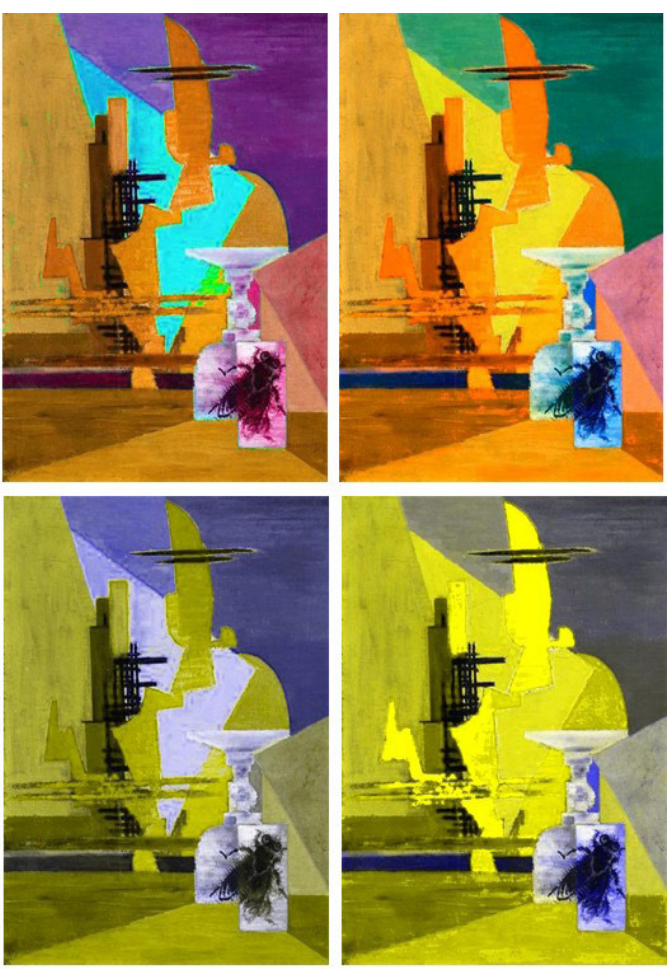

Method 2

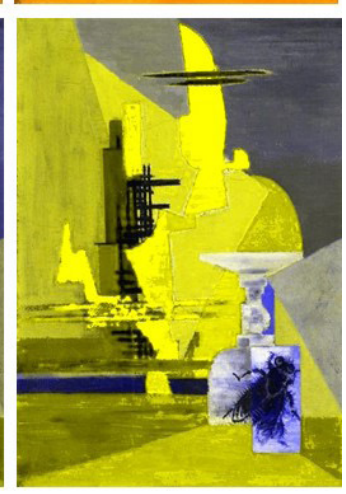

Method 3 
Figure 10. Painting 6: Qualitative comparison for deuteranopia; The original and the recolored paintings are given in the first row, while their respective deuteranope simulations are given in the second row.

In Figure 5, the proposed recolored image uses colors that are more similar to the original image, while enhancing the contrast between the various geometrical objects appearing in the painting. Method 1 and 2 prefer to use greenish hues, while method 3 bluish hues. Next, we investigate the results of the above recoloring as they are reflected in the respective protanope simulated paintings. Staring with Method 3, it is observed that the way the bluish colors are used does not provide efficient contrast enhancement because the protanope viewer cannot distinguish the simulated blue recolored objects from the blue objects that were existed in the original image. Regarding Method 1 and Method 2, similar effects are observed between the simulated green objects and the green objects that were existed in the original painting, in the first place.

In Figure 6, Method 1 prefers reddish and bluish colors to carry out the recoloring process; Method 2 uses brownto-orange and blue hues; while Method 3 persists in using bluish colors. Note that Method 1 and Method 2 significantly change the wall in the painting's background, although that image area is well distinguished by the dischromat, as shown in the protanope simulation of the original painting.

In Figure 7, Method 3 employs again bluish colors without changing a lot the original painting. Method 2, obtains an efficient recoloring of the old man's coat, but it substantially changes the colors for the grass and the tree, while Method 1 prefers the green color to perform the recoloring process. Contrary to the above methods, the proposed algorithm uses colors similar to the colors of the original image (see the old man's coat), while the protanope simulated painting maintains the contrast between the painting's objects.

Finally, similar results are obtained in Figures 8-10 that concern the paintings 4, 5, 6 for the deuteranopia case. In these figures, it can be easily observed that the modified colors of the proposed methods are closer to the original colors, retaining the painting's aesthetics and therefore its naturalness.

\subsection{Subjective evaluation}

In this experiment, eight volunteers evaluated the tested algorithms using pairwise comparisons. It must be noted that the responses were completely anonymous without any personal identification. One of them was protanope, two of them were protanomalous trichromats, and five deuteranomalous trichromats.

As proved in [3], protanopes and protanomalous trichromats show similar behavior in distinguishing colors. The same relation appears to hold for deuteranopes and deuteranomalous trichromats. Thus, we classified the volunteers into two groups namely, Protan and Deutan groups. Protan group included 3 volunteers (i.e., 1 protanope and 2 protanomalous trichromats), while Deutan group included 5 volunteers (i.e., 5 deuteranomalous trichromats).

The tested images were the paintings depicted in Figure 5. For each painting, the original image along with the four recolored images obtained by the four tested methods were displayed and compared by each viewer. The volunteers were asked to answer the next two questions.

Q1: "Which image has the most pleasant contrast?"

Q2: "What is your overall preference?"

For each volunteer and each painting, there are 6 comparisons between the 4 methods. Since 6 paintings are considered, for each volunteer, the number of pairwise comparisons is $6 \times 6=36$. Thus, in total, for each question, there were $3 \times 36=108$ pairwise comparisons for the Protan group and $5 \times 36=180$ pairwise comparisons for the Deutan group.

The above pairwise comparisons were further evaluated by measuring the individuals' preference orderings for the stimuli taken from the set of discrete binary choices. To perform that task, the corresponding average preference scores were estimated by using Thurstone's law of comparative judgement [39]. This law has proven to be very efficient in performing the ranking of items based on subjective choices of individuals.

In view of the above-mentioned two questions, the resulting average preference scores along with the respective $95 \%$ confidence intervals of the pairwise comparisons between the four methods with respect to Protan and Deutan groups are depicted in Figure 11. The analysis illustrated in this figure directly indicates the superiority of the proposed method, supporting the results obtained in the previous sections of the experimental analysis. In addition, it is evident that the competition between Method 1 and Method 3 is strong, giving in some cases better results for the former, and in other cases better results for the latter. 
In summary, the results of the subjective evaluation indicate that the proposed recoloring algorithm obtains effective contrast enhancement while maintaining a pleasant natural appearance of the recolored painting.
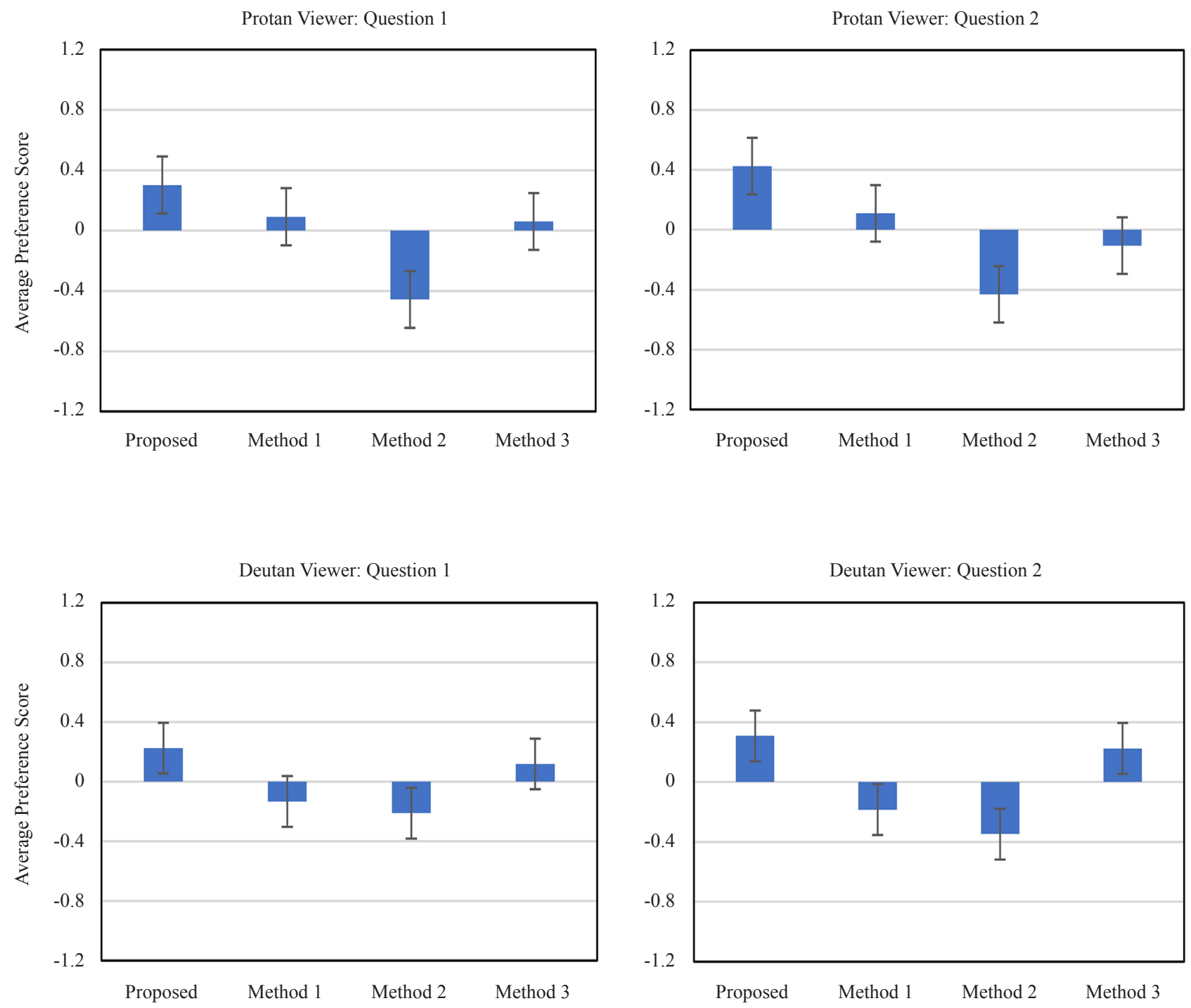

Figure 11. Average preference scores (blue column bars) and the corresponding $95 \%$ confidence intervals for the Protan and Deutan groups, regarding the two questions.

\section{Conclusions}

In this paper, we described a methodology able to appropriately recolor art paintings, alleviating the effects of two types of dichromacy namely, protanopia and deuteranopia. The method is implemented in the RGB color space and involves four steps applied in sequence. The first step reduces the number of colors used in the subsequent steps by obtaining a clustering of the input image pixels into a number of representative colors. In the second step, a standard algorithm is used to elaborate the above representative colors and transform them into colors perceived correctly by the protanopes or deuteranopes. Representative colors that have not been changed much remain intact, while those that have been changed a lot are recolored, because they are confused by the color-blind. That recoloring process takes place in step 3, and it is carried out in terms of an optimization-based daltonization procedure. Finally, all input image pixels belonging to the clusters defined by the changed representative colors are appropriately recolored. The proposed algorithm was tested over a set of art paintings using quantitative and qualitative analysis, where it was compared to 
other two similar approaches in terms of two performance indices. The main results indicate that the method is very competitive to the other methods, obtaining natural recoloring and maintaining the aesthetic of the original paintings.

Future efforts involve: (a) the real time practical implementation of the methodology in the form of the smartphone application, where the color-blind user during his/her visit to a museum will be able to take a photo of a painting and the application will generate and display the modified painting, and (b) the use of more sophisticated methods, like deep neural networks, to improve the quality of the recolored paintings.

\section{Conflicts of Interest}

The authors declare no conflict of interest.

\section{Acknowledgements}

The authors would like to thank the anonymous reviewers for their effort to provide their valuable comments.

\section{References}

[1] Moreira H, Alvaro L, Melnikova A, Lillo J. Colorimetry and dichromatic vision. In: Travieso-Gonzalez C. (ed.) Colorimetry and Image Processing. London, UK: IntechOpen; 2018. p. 2-21.

[2] Fairchild MD. Color appearance models. West Sussex, UK: John Wiley and Sons; 2005.

[3] Smith VC, Pokorny J. Color matching and color discrimination. In: Shevell S. (ed.) The Science of Color. Oxford, UK: Elsevier; 2003. p. 103-148. Available from: https://doi.org/10.1016/B978-044451251-2/50004-0.

[4] Stockman A, Sharpe LT. The spectral sensitivities of the middle-and long-wavelength-sensitive cones derived from measurements in observers of known genotype. Vision Research. 2000; 40(13): 1711-1737. Available from: https:// doi.org/10.1016/s0042-6989(00)00021-3.

[5] Ribeiro M, Gomes AJ. Recoloring algorithms for colorblind people: A survey. ACM Computing Surveys. 2019; 52(4): 1-37.

[6] Tsekouras GE, Rigos A, Chatzistamatis S, Tsimikas J, Kotis K, Caridakis G, et al. A novel approach to image recoloring for color vision deficiency. Sensors. 2021; 21: 2740. Available from: https://www.mdpi.com/14248220/21/8/2740 [Accessed 13th September 2021].

[7] Lin HY, Chen LQ, Wang ML. Improving discrimination in color vision deficiency by image re-coloring. Sensors. 2019; 19: 2250. Available from: https://www.mdpi.com/1424-8220/19/10/2250 [Accessed 15th September 2021].

[8] Chatzistamatis S, Rigos A, Tsekouras GE. Image recoloring of art paintings for the color blind guided by semantic segmentation. Proceedings of 21 st International Conference on Engineering Applications of Neural Networks (EANN 2020), 5-7 June 2020. Halkidiki, Greece. Switzerland: Springer Nature; 2020. p. 261-273.

[9] Wong A, Bishop W. Perceptually-adaptive color enhancement of still images for individuals with dichromacy. Proceedings of the 21 $1^{\text {st }}$ IEEE Canadian Conference on Electrical and Computer Engineering, 4-7 May 2018. Niagara Fall, Canada. Piscataway, NJ, USA: IEEE; 2008. p. 2027-2031.

[10] Hassan MF, Paramesran R. Naturalness preserving image recoloring method for people with red-green deficiency. Signal Processing: Image Communication. 2017; 57: 126-133.

[11] Rigos A, Chatzistamatis S, Tsekouras GE. A systematic methodology to modify color images for dichromatic human color vision and its application in art paintings. International Journal of Advanced Trends in Computer Science and Engineering. 2020; 9(4): 5015-5025.

[12] Johnston-Feller R. Color science in the examination of museum objects: Nondestructive procedures. Los Angeles, CA: Getty Conservation Institute. 2001. Available from: http://hdl.handle.net/10020/gci_pubs/color_science [Accessed 17th September 2021].

[13] Ho GW. Color, vision, and art: Teaching, learning, and making art with color blind awareness. MsC Thesis, University of Florida. 2014. Available from: https://www.hoyangfineart.com/uploads/1/6/5/2/16529980/ho_ capstonefinaldec3.pdf [Accessed 17th September 2021].

[14] Marmor MF, Lanthony P. The dilemma of color deficiency and art. Survey of Ophthalmology. 2001; 45(5): 407- 
414.

[15] Choi J, Lee J, Moon H, Yoo SJ, Han D. Optimal color correction based on image analysis for color vision deficiency. IEEE Access. 2019; 7: 154466-154479. Available from: https://doi.org/10.1016/s0039-6257(00)001922.

[16] Kuhn GR, Oliveira MM, Fernandes LAF. An efficient naturalness-preserving image-recoloring method for dichromats. IEEE Transactions on Visualization and Computer Graphics. 2008; 14(6): 1747-1754. Available from: https://doi.org/10.1109/TVCG.2008.112.

[17] Wang X, Zhu Z, Chen X, Go K, Toyoura M, Mao X. Fast contrast and naturalness preserving image recolouring for dichromats. Computer \& Graphics. 2021; 90: 19-28.

[18] Kang SK, Lee C, Kim CS. Optimized color contrast enhancement for dichromats using local and global contrast. Proceedings of the 2020 IEEE International Conference on Image Processing (ICIP 2020), 25-28 October 2020. Abu Dhabi, UAE. Piscataway, NJ, USA: IEEE; 2020. p. 1048.

[19] Kovalev V, Petrou M. Optimising the choice of colours of an image database for dichromats. Lecture Notes in Artificial Intelligence. 2005; 3587: 456-465.

[20] Zhu Z, Toyoura M, Go K, Fujishiro I, Kashiwagi K, Mao X. Naturalness and information-preserving image recoloring for red-green dichromats. Signal Processing: Image Communication. 2019; 76: 68-80. Available from: https://doi.org/10.1016/j.image.2019.04.004.

[21] Bao J, Wang Y, Ma Y, Gu X. Re-coloring images for dichromats based on an improved adaptive mapping algorithm. Proceedings of the 2008 IEEE International Conference on Audio, Language and Image Processing, 7-9 July 2008. Shanghai, China. Piscataway, NJ, USA: IEEE; 2008. p. 152-156.

[22] Lai CL, Chang SW. An image processing based visual compensation system for vision defects. Proceedings of the 5th IEEE International Conference on Intelligent Information Hiding and Multimedia Signal Processing (IIHMSP 2009), 12-14 September 2009, Kyoto, Japan. Piscataway, NJ, USA: IEEE; 2009. p. 559-562.

[23] Ma Y, Gu X, Wang Y. Color discrimination enhancement for dichromats using self-organizing color transformation. Information Sciences. 2009; 179: 830-843.

[24] Li H, Zhang L, Zhang X, Zhang M, Zhu G, Shen P, et al. Color vision deficiency datasets \& recoloring evaluation using GANs. Multimedia Tools and Application. 2020; 79: 27583-27614.

[25] Xu Q, Zhang X, Zhang L, Zhu G, Song J, Shen P. An efficient recoloring method for color vision deficiency based on color confidence and difference. Proceedings of the $2^{\text {nd }}$ CCF Chinese Conference on Communication in Computer and Information Science (CCCV 2017), October 11-14 2017, Tianjin, China. Singapore: Springer Nature; 2017. p. 270-281.

[26] Bonacin R, Cesar J, Araujo R. An ontology-based framework for improving color vision deficiency accessibility. Universal Access in the Information Society. 2021. Available from: https://doi.org/10.1007/s 10209-021-00791-6.

[27] Ching SL, Sabudin M. Website image colour transformation for the colour blind. Proceedings of the $2^{\text {nd }}$ IEEE International Conference on Computer Technology and Development, 2-4 November 2010, Cairo, Egypt. Piscataway, NJ, USA: IEEE; 2010. p. 255-259.

[28] Nakauchi S, Onouchi T. Detection and modification of confusing color combinations for red-green dichromats to achieve a color universal design. Color Research \& Application. 2008; 33: 203-211. Available from: https://doi. org/10.1002/col.20404.

[29] Huang JB, Chen CS, Jen TS, Wang SJ. Image recolorization for the color blind. Proceedings of the 2009 IEEE International Conference on Acoustics, Speech and Signal Processing (ICASSP 2009), 19-24 April 2009, Tapei, Taiwan. Piscataway, NJ, USA: IEEE; 2009. p. 1161-1164.

[30] Li J, Feng X, Fan H. Saliency-based image correction for colorblind patients. Computational Visual Media. 2020; 6(2): 169-189.

[31] Celebi ME, Kingravi HA, Vela PA. A comparative study of efficient initialization methods for the k-means clustering algorithm. Expert Systems with Applications. 2013; 40(1): 200-210.

[32] De Oliveira JV, Pedryz W. Advances in fuzzy clustering. West Sussex, UK: John Wiley and Sons; 2007.

[33] Hoppner F, Klawonn F, Kruse R, Runkler T. Fuzzy cluster analysis: Methods for classification, data analysis and image recognition. West Sussex, UK: John Wiley and Sons; 2000.

[34] Niros AD, Tsekouras GE. A novel training algorithm for RBF neural network using a hybrid fuzzy clustering approach. Fuzzy Sets and Systems. 2012; 193: 62-84.

[35] Vienot F, Brettel H, Ott L, M' Barek AB, Mollon JD. What do color-blind people see. Nature. 1995; 376: $127-128$.

[36] Vienot F, Brettel H, Mollon JD. Digital video colourmaps for checking the legibility of displays by dichromats. Color Research \& Application. 1999; 24: 243-252. Available from: https://doi.org/10.1002/(SICI)15206378(199908)24:4<243::AID-COL5>3.0.CO;2-3. 
[37] Price KV, Storn RM, Lampinen JA. Differential evolution: a practical approach to global optimization. Berlin, Germany: Springer; 2005.

[38] Zhang L, Zhang L, Mou X, Zhang D. FSIM: A feature similarity index for image quality assessment. IEEE Transactions on Image Processing. 2011; 20(8): 2378-2386. Available from: https://doi.org/10.1109/ TIP.2011.2109730.

[39] Thurstone LL. A law of comparative judgement. Psychology Review. 1927; 34: 273-286.

[40] WikiArt. Available from: https://www.wikiart.org [Accessed on 5 December 2021]. 